\title{
Pompéi, Porta Ercolano : organisation, gestion et transformations d'une zone suburbaine
}

Campagne 2016

Sandra Zanella, Laetitia Cavassa, Nicolas Laubry, Nicolas Monteix, Guilhem Chapelin, Arnaud Coutelas, Audrey Delvigne Ryrko, Mélanie Errera, Léa Gerardin, Bastien Lemaire, Raphael Macario, Florien Ortis, Vincenzo Pellegrino et Géraldine Sachau-Carcel

\section{(2) OpenEdition}

Édition électronique

URL : http://journals.openedition.org/cefr/1676

DOI : $10.4000 /$ cefr. 1676

ISSN : 2282-5703

Éditeur

École française de Rome

Référence électronique

Sandra Zanella, Laetitia Cavassa, Nicolas Laubry, Nicolas Monteix, Guilhem Chapelin, Arnaud Coutelas , Audrey Delvigne Ryrko, Mélanie Errera, Léa Gerardin, Bastien Lemaire, Raphael Macario, Florien Ortis, Vincenzo Pellegrino et Géraldine Sachau-Carcel, « Pompéi, Porta Ercolano : organisation, gestion et transformations d'une zone suburbaine ", Chronique des activités archéologiques de l'École française de Rome [En ligne], Les cités vésuviennes, mis en ligne le 08 mars 2017, consulté le 11 septembre 2019. URL : http://journals.openedition.org/cefr/1676 ; DOI : 10.4000/cefr.1676

Ce document a été généré automatiquement le 11 septembre 2019.

(c) École française de Rome 


\title{
Pompéi, Porta Ercolano : organisation, gestion et transformations d'une zone suburbaine
}

\author{
Campagne 2016
}

Sandra Zanella, Laetitia Cavassa, Nicolas Laubry, Nicolas Monteix, Guilhem Chapelin, Arnaud Coutelas, Audrey Delvigne Ryrko, Mélanie Errera, Léa Gerardin, Bastien Lemaire, Raphael Macario, Florien Ortis, Vincenzo Pellegrino et Géraldine Sachau-Carcel

\section{NOTE DE L'AUTEUR}

Cette opération a été financée par le Centre Jean Bérard, par l'École française de Rome, par le Centre Camille Jullian (UMR 7299), et a bénéficié de fonds privés dans le cadre d'un mécénat de la part de deux sociétés françaises : cmD2, Art et Luxe, Artfusion et des financements privés.

L'équipe était composée de Sandra Zanella (Labex Archimede - Université Montpellier III, UMR 5140 Archéologie des Sociétés Méditerranéennes / UMR 8546 AOROC), Laëtitia Cavassa (Aix Marseille Univ, CNRS, Minist Culture \& Com, CCJ, Aix-en-Provence, France), Nicolas Laubry (Université de Paris Est-Créteil, CRHEC) et Nicolas Monteix (Institut Universitaire de France-Université de Rouen), Sanna Aho (Archéologue), Henri Duday (UMR 5199 PACEA, Équipe A3P-Anthropologie des populations passées et présentes, Université de Bordeaux), Gabriele Salvatore Boi (archéologue, Université de Bologne), Antoine Boisson (archéologue, Université de Montpellier), Guilhem Chapelin (architecte, Centre Jean Bérard), Sevérine Corbeel (doctorante de l'Université de Montpellier), Arnaud Coutelas (Chargé d'études-responsable d'opération, ArkeMine, UMR 8546 AOROC), Saverio de Rosa (numismate), Audrey Delvigne Ryrko (archéologue), Anaïs Denysiak (archéologue), Christine Durand (photographe, Aix Marseille Univ, CNRS, Minist Culture 
\& Com, CCJ, Aix-en-Provence, France), Mélanie Errera (archéologue, Université de Montpellier), François Fouriaux (archéologue, topographe, Eveha), Léa Gerardin (archéologue, Hadès), Giorgia La Placa (archéologue, Université de Bologne), Loïc Lefévre (archéologue, Université de Rouen), Bastien Lemaire (doctorant à l'Université Paul-Valéry Montpellier III), Pierpaolo Lippolis (archéologue, Université de Bologne), Fabrizio Luisani (archéologue, Université de Bologne), Raphael Macario (archéologue, Hadès), Sandrine Mencarelli (architecte), Agnès Oboussier (restauratrice, (Aix Marseille Univ, CNRS, Minist Culture \& Com, CCJ, Aix-en-Provence, France), Florien Ortis (archéologue, Université de Montpellier), Frédéric Pauvarel (photographe), Vincenzo Pellegrino (doctorant à l'Université Paul-Valéry Montpellier III), Rémi Rêve (archéologue, Aix-Marseille Université), Carla Rosa (étudiante à l'université de Fisciano-Salerno), Géraldine SachauCarcel (UMR 5199 PACEA, Équipe A3P-Anthropologie des populations passées et présentes, Université de Bordeaux), Ophélie Vauxion (spécialiste des enduits peints). Nous remercions également Bruno Desachy, auteur du Stratifiant, outil d'aide au raisonnement chronologique de terrain qui a démontré sa grande utilité au sortir de la fouille.

Nous remercions la Soprintendenza Pompei pour nous avoir permis de travailler dans les meilleures conditions. Nous remercions tout particulièrement le Professeur Massimo Osanna, directeur général de la Soprintendenza Pompei, la dott.ssa Grete Stefani, directrice du site, la dott.ssa Annalisa Capurso, fonctionnaire archéologue responsable de notre secteur, arch.tta Annamaria Mauro, fonctionnaire architecte, Vincenzo Sabini, assistant technique, Domenico Busiello responsable des dépôts.

1 Il s'agit de la cinquième campagne de fouilles d'un projet plus large faisant partie du programme de recherche quinquennal de l'École française de Rome, mené sous la direction de Sandra Zanella, Laëtitia Cavassa, Nicolas Laubry et Nicolas Monteix et intitulé « Organisation, gestion et transformations d'une zone suburbaine : le secteur de la Porte d'Herculanum à Pompéi, entre espace funéraire et commercial ». Cette recherche s'intéresse à la zone de la porta Ercolano, située immédiatement à l'extérieur des murs de la colonie. Le projet est réalisé dans le cadre d'une concession de fouilles pluriannuelle (2012-2016) accordée par le Ministero per i Beni e le attività culturali. La campagne de cette année, s'est déroulée sur deux mois, du 15 mai au 15 juillet 2016 et du 20 septembre au 2 octobre.

2 Cette campagne visait à recueillir des informations sur certains des aspects importants du projet: les activités artisanales qui se déroulaient dans les boutiques et l'évolution urbaine du secteur. Plusieurs boutiques et secteurs du portique et du trottoir longeant la via dei Sepolcri ${ }^{1}$ et la via Superior ont fait l'objet de sondages et nettoyages (fig. 1). 
Fig. 1 - Plan d'ensemble du secteur avec en évidence les zones intéressées par des opérations de nettoyage et fouille en 2016.

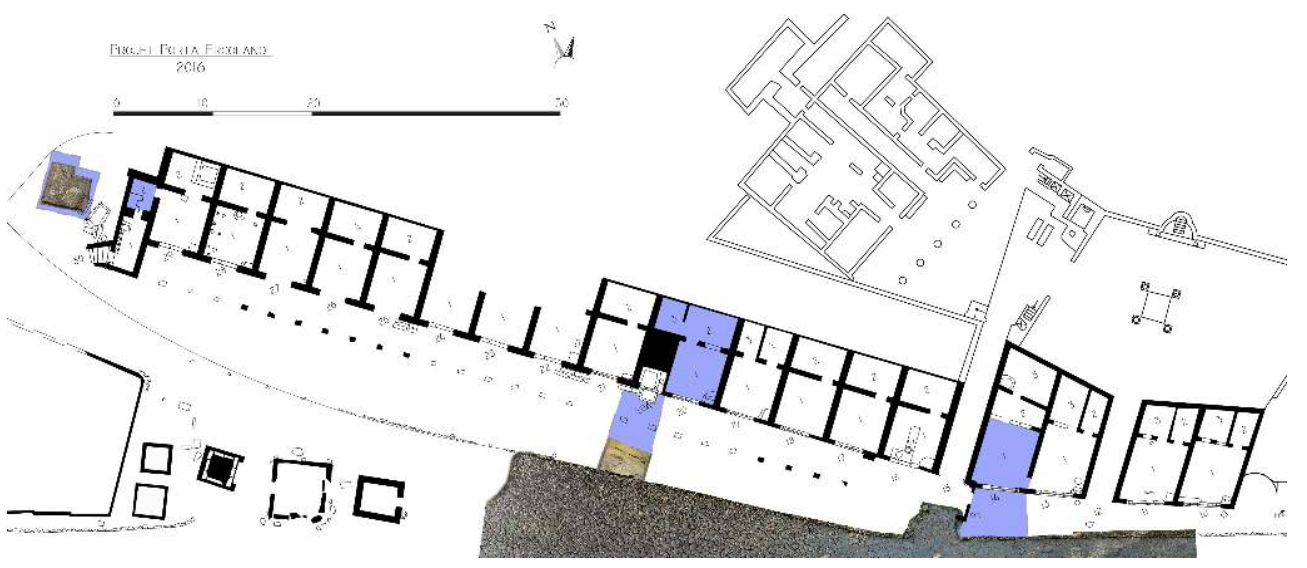

Élaboration S. Zanella-EFR.

C'est le cas de la boutique N14, dont la fouille avait été commencée en 2015 ; du trottoir en avant de cette boutique (indiqué comme T1013) ; de la boutique N20 ; du portique et du trottoir en avant de la fontaine $\mathrm{N} 20 \mathrm{a}$ (PN2021) ; de la pièce N30/2 et de la sépulture N32a à l'extrémité occidentale du portique. L'étude du bâti a été également terminée sur l'ensemble des boutiques.

\section{L'étude du bâti des boutiques au nord de la via dei Sepolcri}

3 L'étude du bâti des boutiques en façade de la villa delle Colonne a mosaico a porté sur les structures maçonnées, sur leurs contacts, sur les techniques et les matériaux de construction. Les observations des mortiers ont été réalisées in situ par A. Coutelas, à partir de l'aspect de surface et de celui au cœur de la maçonnerie. Ce travail a été complété par un enregistrement des unités stratigraphiques murales (USM) des boutiques N10-N14, N20 et N29. Au nord des boutiques N15 à N29, les observations des structures bâties ont été limitées par l'absence de dégagement, par la présence du talus et d'une végétation dense.

4 Deux grands ensembles se dégagent de part et d'autre du couloir d'accès à la villa N15 (fig. 2). Ils se distinguent par les modules de leurs plans et par les mortiers employés dans la construction. Le premier (ensemble 1), situé à l'ouest du couloir, comprend les boutiques N16-N29, qui ont connu deux états. 
Fig. 2 - Plan des boutiques en dehors de la porta Ercolano avec les différentes interventions effectuées sur le bâti mises en évidence.

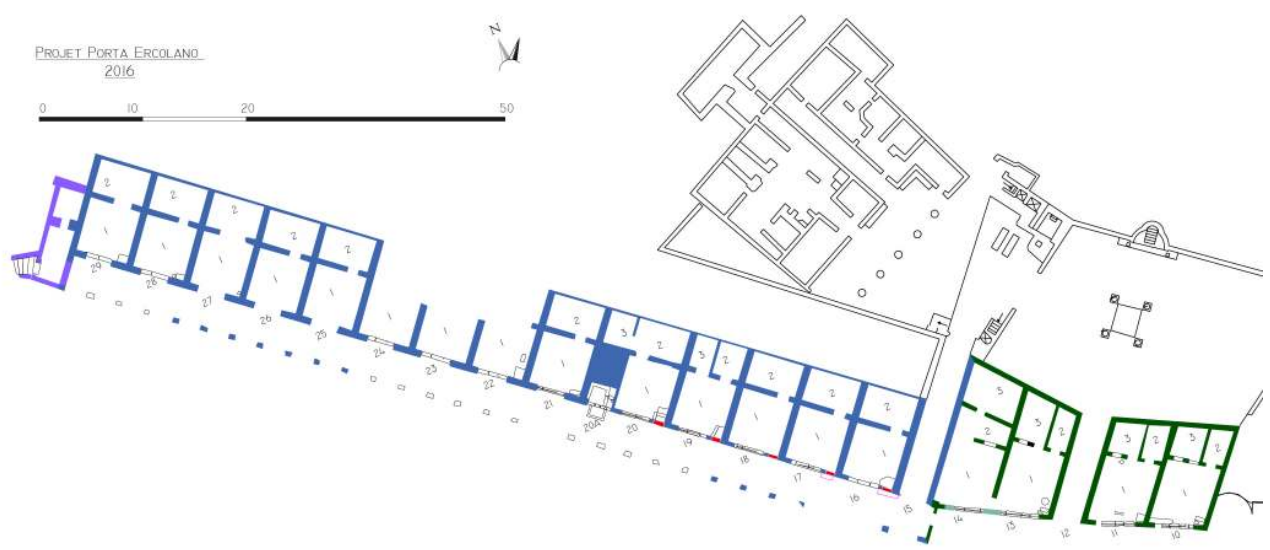

Élaboration : S. Zanella-EFR.

Leur module, divisé en deux pièces, est régulier. Leur rez-de-chaussée fut dans un premier temps recouvert d'un plancher dont l'emprise coïncidait avec la pièce nord, séparée par un mur de refend. L'escalier d'accès donnait sur une porte ouverte sur le portique - aménagements observables au moins pour N16-N20 et N29 (fig. 3). Les façades sont en opus incertum en moellons de basalte, avec une différence pour les accès principaux entre les boutiques à l'est et à l'ouest de la fontaine N20a. Pour les premières, les montants sont en calcaire du Sarno, tandis qu'ils sont en opus vittatum mixtum pour les secondes, avec emploi de tuf jaune des «Champs Phlégréens » (N25-N29). Ces montants furent réalisés dans une phase successive de réfection de la façade comme le révèlent des coups de sabre verticaux et l'usage de mortier différent (jaune-brun avec gros nodules de chaux).

Fig. 3 - Vue de la porte bouchée et de la banquette en façade de la boutique N17.

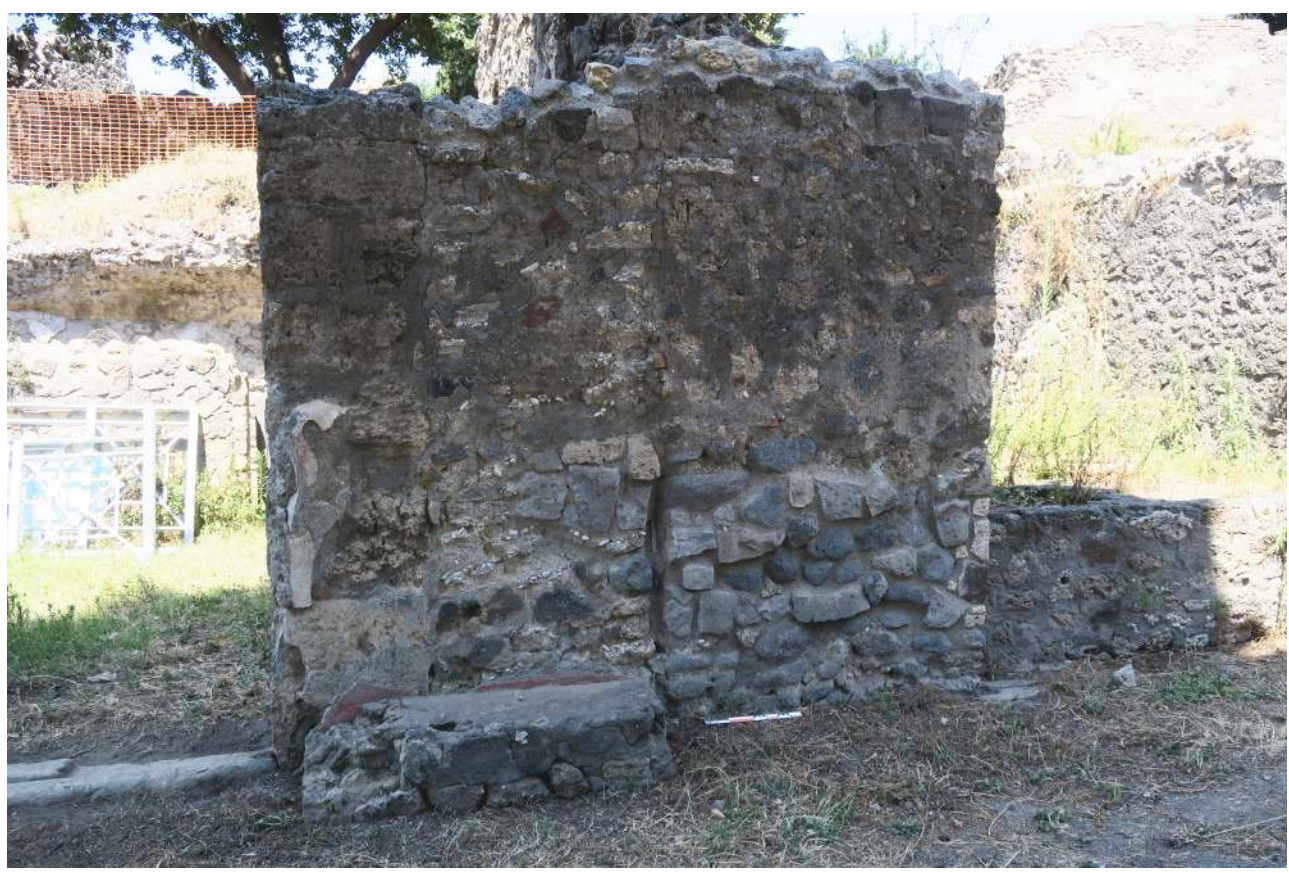

Cliché : G. Chapelin-EFR.

Chronique des activités archéologiques de l'École française de Rome , Les cités vésuviennes 
La seconde phase se traduit par l'obstruction des portes piétonnes (fig. 2 - en rouge), ainsi que par l'installation de banquettes devant les boutiques $\mathrm{N} 16$ et N17, tandis que des voûtes en berceau furent édifiées dans la pièce septentrionale des boutiques, avec des matériaux (cruma et éléments de récupération) et un mortier homogènes. Des amphores engagées dans la maçonnerie donnent un horizon chronologique du $\mathrm{I}^{\mathrm{er}} \mathrm{s}$. de notre ère. La plupart des boutiques témoignent enfin d'une campagne de reprise de l'ensemble des enduits.

Dans cet ensemble, la fontaine (N20a) se démarque par l'exiguïté de son plan, qui n'est pas le résultat de la modification, mais fut originellement conçue de la sorte. L'espace comporte trois bassins (fig. 4), réalisés à partir de diverses techniques de construction et matériaux (terre cuite, bloc de calcaire du Sarno).

Fig. 4 - Plan et coupe de la fontaine N20a.

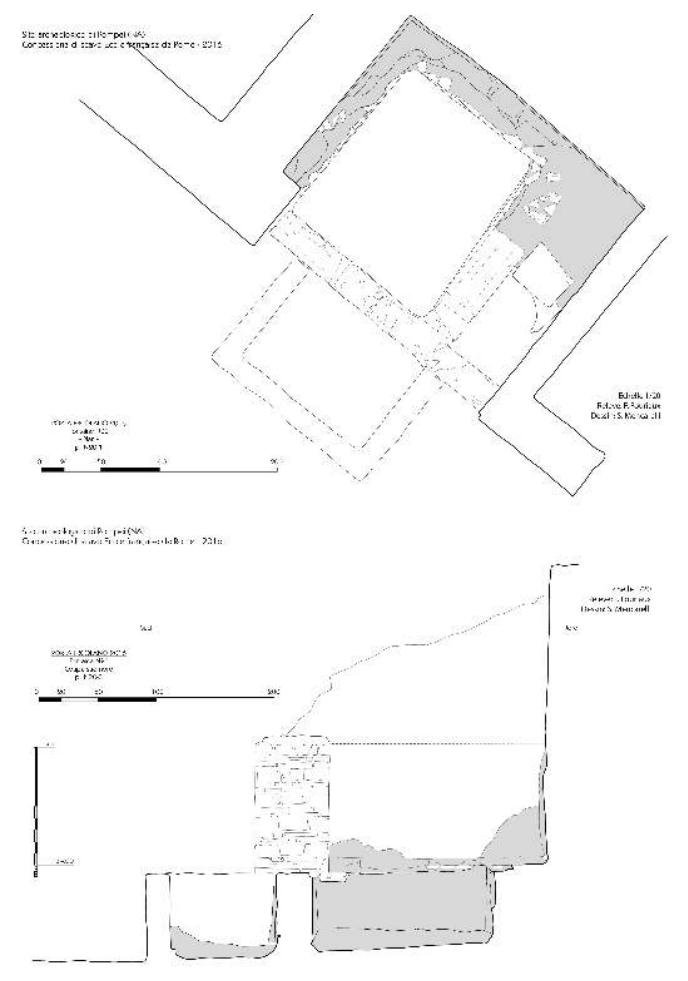

Échelle : $1 / 20$.

Relevé et dessin : Fr. Fouriaux / S. Mencarelli-EFR.

Le bassin le plus méridional, qui est un ajout, déborde l'alignement de la façade des boutiques. De même, la boutique N20 se distingue par la singularité de son plan en L, puisqu'elle a bénéficié au nord de l'adjonction d'une pièce dans l'axe de la fontaine N20a, qui lui est solidaire dès l'origine. Dans l'état initial, les pièces 2 et 3 de cette boutique n'étaient pas séparées. Plusieurs aménagements postérieurs à l'état initial ont été observés, dont l'installation d'une canalisation venant couper la fosse de coulée de bronze de la pièce 3 (cf. infra).

7 L'accès à la villa delle Colonne a mosaico (N15) partage avec l'ensemble 1 la technique de construction et présente un mortier similaire - mais non identique - indiquant qu'ils furent mis en place au même moment. Ce couloir marque la transition avec l'ensemble 2, qui apparaît comme le résultat d'une phase d'aménagement ultérieure du secteur en 
phase avec le réaménagement du secteur oriental de la villa. Le mortier notamment (jaune-brun avec fréquents nodules de chaux) se distingue de celui des boutiques N16N29. Des murs périphériques en petits moellons de basalte, avec finition en pietra rasa, furent élevés dans un premier temps. La partie haute est en opus incertum de calcaire du Sarno et de cruma. Des fenêtres étaient aménagées dans le mur du nord des boutiques qui disposaient d'un étage, avec latrine dans les angles nord-est de 13 et 14 . Tandis que les murs latéraux sont liés aux murs nord, l'irrégularité de l'orientation de ces derniers résulte du plan de l'extension de la villa. L'ensemble paraît former un tout homogène, issu d'un projet unitaire de réorganisation.

La mise en place des murs divisant les boutiques en plusieurs pièces intervint dans un second temps mais très probablement suivant un projet initial. L'agencement des boutiques N10 et N11 est similaire, avec un escalier à droite et une partition plus tardive de la pièce septentrionale. Les boutiques N13-14, reliées par une porte entre les pièces 1 des deux boutiques, semblent en revanche devoir être considérées comme un même ensemble originel. Les piédroits en tuile furent alors installés en façade. À l'intérieur, l'enduit correspond à un seul état de revêtement. Observable en plusieurs points, la décoration de façade met l'accent sur l'entrée N12 de la villa, flanquée de pilastres de stuc pour en souligner la monumentalité, et elle possède deux états successifs (fig. 5).

Fig. 5 - Vue de l'accès N12 à la villa delle Colonne a mosaico depuis la via dei Sepolcri.

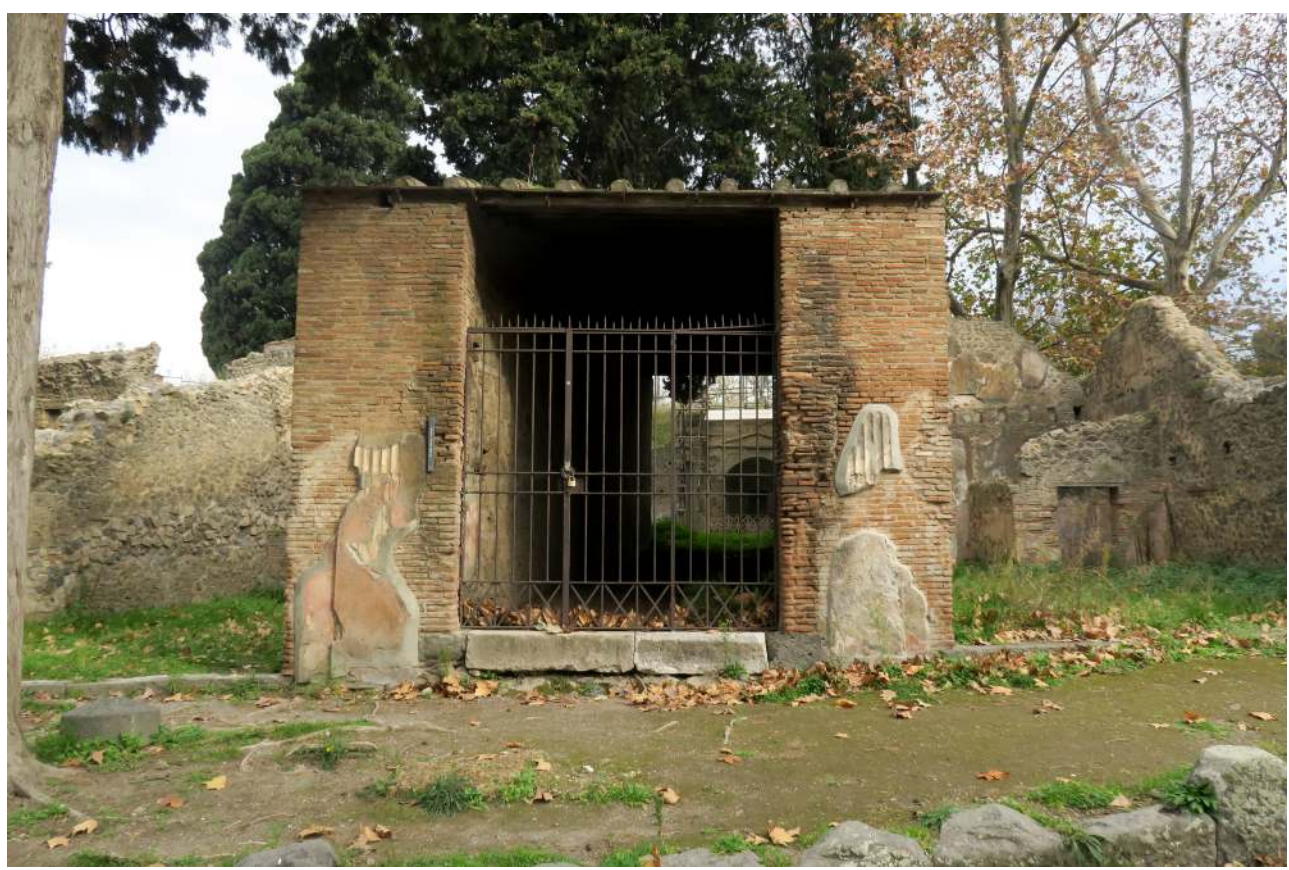

Cliché : G. Chapelin-EFR

Elle reprend celle de la façade orientale de l'arc du portique et l'on remarque, en particulier dans la partie basse, des ornements qui apparaissaient peut-être dans le premier état du décor de ce dernier (palmettes à trois feuilles et fleurs de lotus à deux pétales et pistil, liées par des tiges géométrisées enveloppant la palmette et formant une volute) (fig. 6). 
Fig. 6 - Vue de l'arche d'entré au portique depuis le trottoir en face de N12.

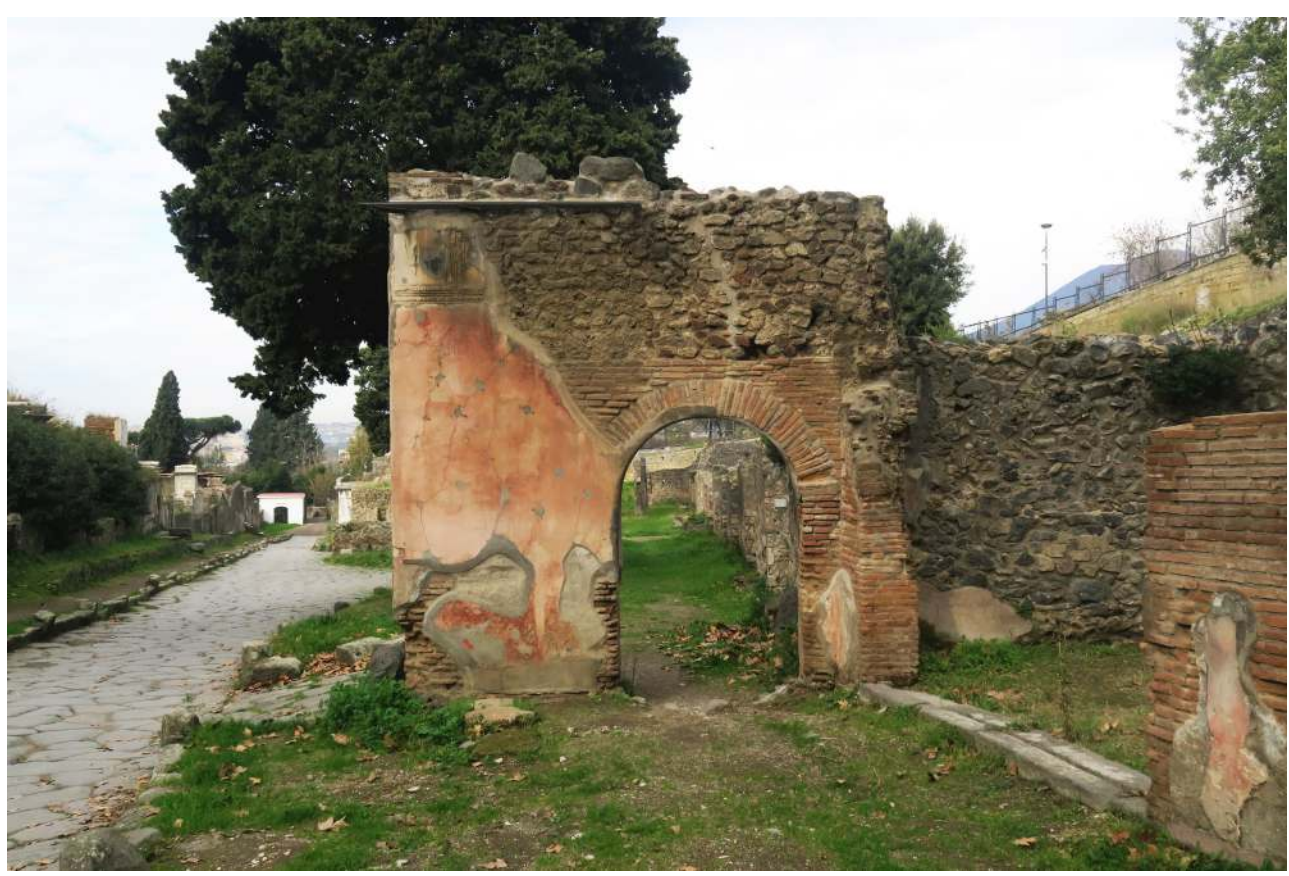

Cliché : G. Chapelin-EFR.

Quant au portique se développant devant l'ensemble 1, il a connu aux moins deux états. $\mathrm{Au}$ niveau de la limite orientale, la partie méridionale du mur séparant N14 et N15, en forme de $\mathrm{T}$, construite en blocs de calcaire du Sarno et prolongée par un piédroit, a servi de départ pour un premier arc, mal conservé, à claveaux en pierre (fig. 2). Dans un second temps, une autre arcade fut édifiée, décalée légèrement vers l'est, reposant sur des montants en opus testaceum et construite avec des fragments de tuiles. La partie supérieure est en opus incertum et surmonte une rangée de trous pour des solives, qui indiquent la présence d'un étage. L'arche était ornée en façade de stucs et d'enduit richement décoré.

Face aux boutiques N17 et N18, deux arches et le départ d'une troisième sont conservées. L'écartement entre les piliers n'est pas totalement régulier. Ces derniers appartiennent à la seconde phase du portique, réalisée partiellement en opus testaceum, avec des fragments de tuiles de production locale liés par un mortier gris à jaunâtre, assez terreux, à sable fin et quelques nodules de chaux. Au niveau des boutiques N25-N26, les vestiges correspondent en revanche au premier état (fig. 7). 
Fig. 7 - Arcade du portique en correspondance des boutiques N25-27.

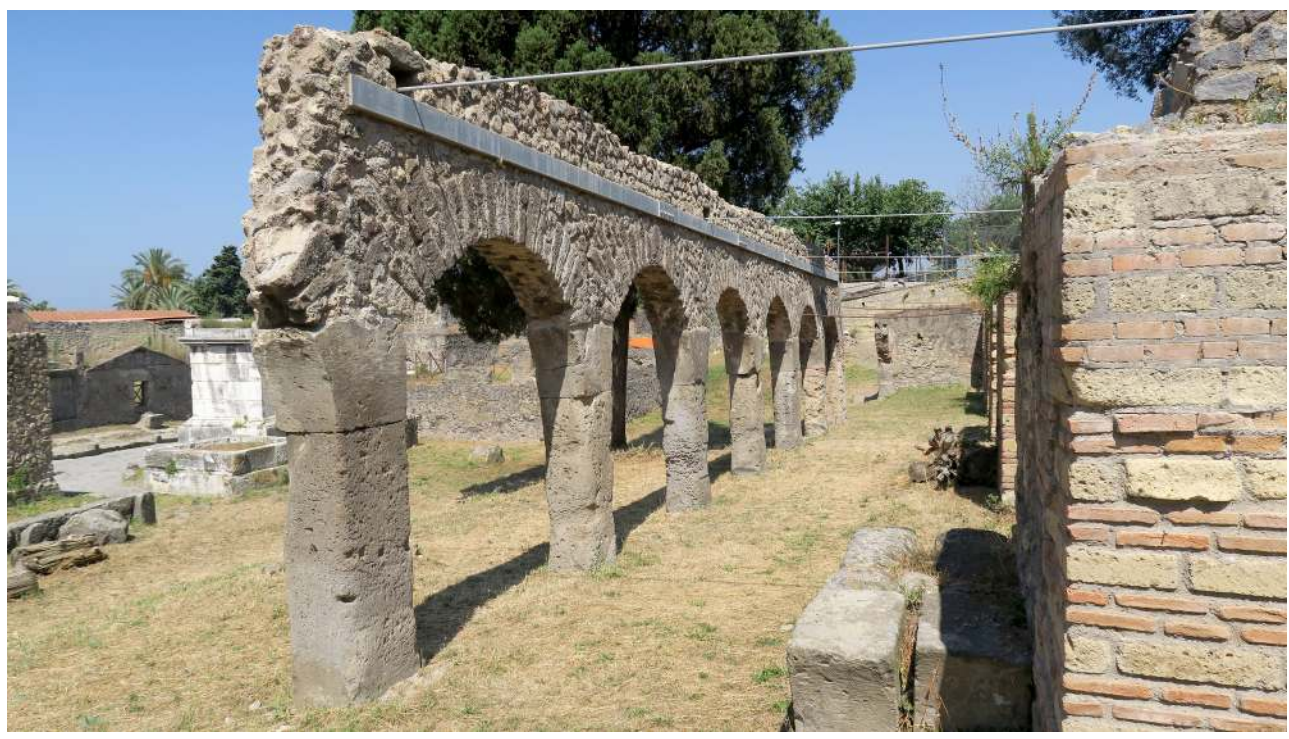

Cliché : G. Chapelin-EFR

Les piliers sont en blocs de tuf de Nocera, avec pour certains des traces de taille ou de récupération. Les claveaux sont aussi en tuf ou en calcaire du Sarno. Le mortier est celui des boutiques de l'ensemble 1, et le lien avec celles-ci est confirmé par le rythme des piliers, en accord avec celui des murs latéraux des boutiques. Le tout était sans doute enduit. Au niveau de la boutique N27, les deux piliers sont en calcaire du Sarno, tandis que les deux avant-derniers portent 2 à 5 assises de terres cuites entre le bloc et l'imposte, le dernier étant réalisé en massif de terres cuites.

Enfin, dans la limite occidentale, face à N29, le dernier pilier présente les mêmes caractéristiques que les précédents, son imposte formant un arc pour le raccord, difficile à lire en raison des restaurations, avec la façade de la boutique. Il n'est cependant pas lié au mur ouest. Cette arche a été bouchée par un parement réalisé avec différents matériaux, dont des fragments de sol à galets. Un jour en archère est aménagé au sommet de ce bouchage, avec la partie la plus étroite côté portique (fig. 8). 
Fig. 8 - Bouchage de l'arche ouest du portique vue depuis l'est.

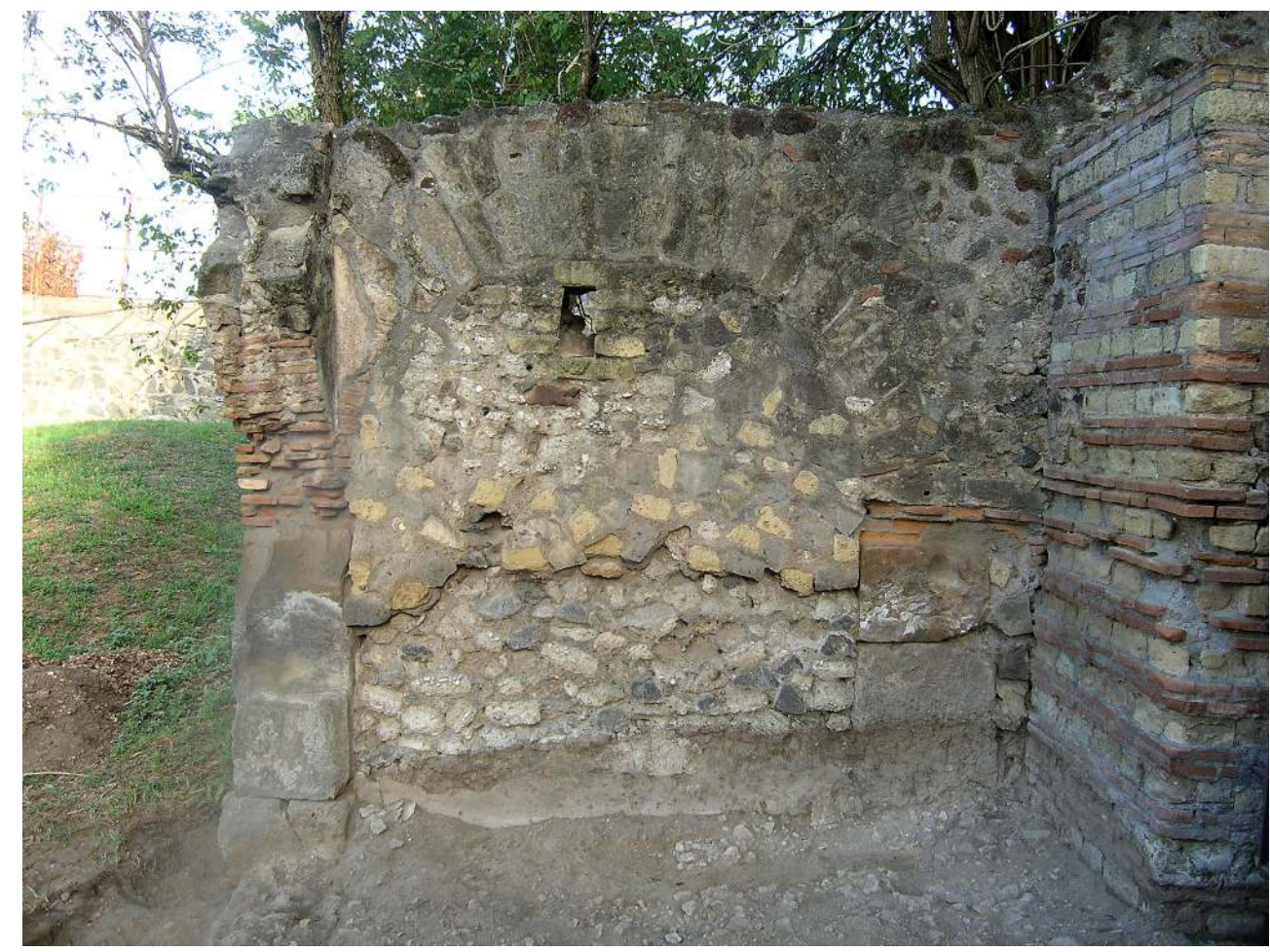

Cliché : G. Chapelin-EFR.

12 L'étude du bâti précise et confirme le lien étroit existant entre la villa, les boutiques et le portique, non seulement, de manière évidente, pour l'ensemble 2, mais aussi manifestement pour l'ensemble 1, suggérant donc à chaque fois des entreprises concertées.

\section{Les sondages dans le portique et le trottoir}

Deux sondages, implantés l'un entre la limite du trottoir de la via dei Sepolcri, en façade de la boutique N14, et l'autre en avant de la fontaine N20a jusqu'au trottoir, nous ont permis d'observer des niveaux correspondant à des voies antérieures à l'implantation du portique et des boutiques, ainsi que leurs phases de réaménagement consécutives (fig. 1).

Devant la boutique N14, trois tranchées modernes, implantées très probablement dans le cadre des travaux de gestion des eaux du site effectués durant les années 1980-1990², ont détruit une partie de la stratigraphie, nous interdisant toute vision cohérente des niveaux anciens (fig. 9). 
Fig. 9 - Plan des tranchées modernes interceptées par notre sondage entre la boutique 14 et la limite du trottoir de la via dei Sepolcri.

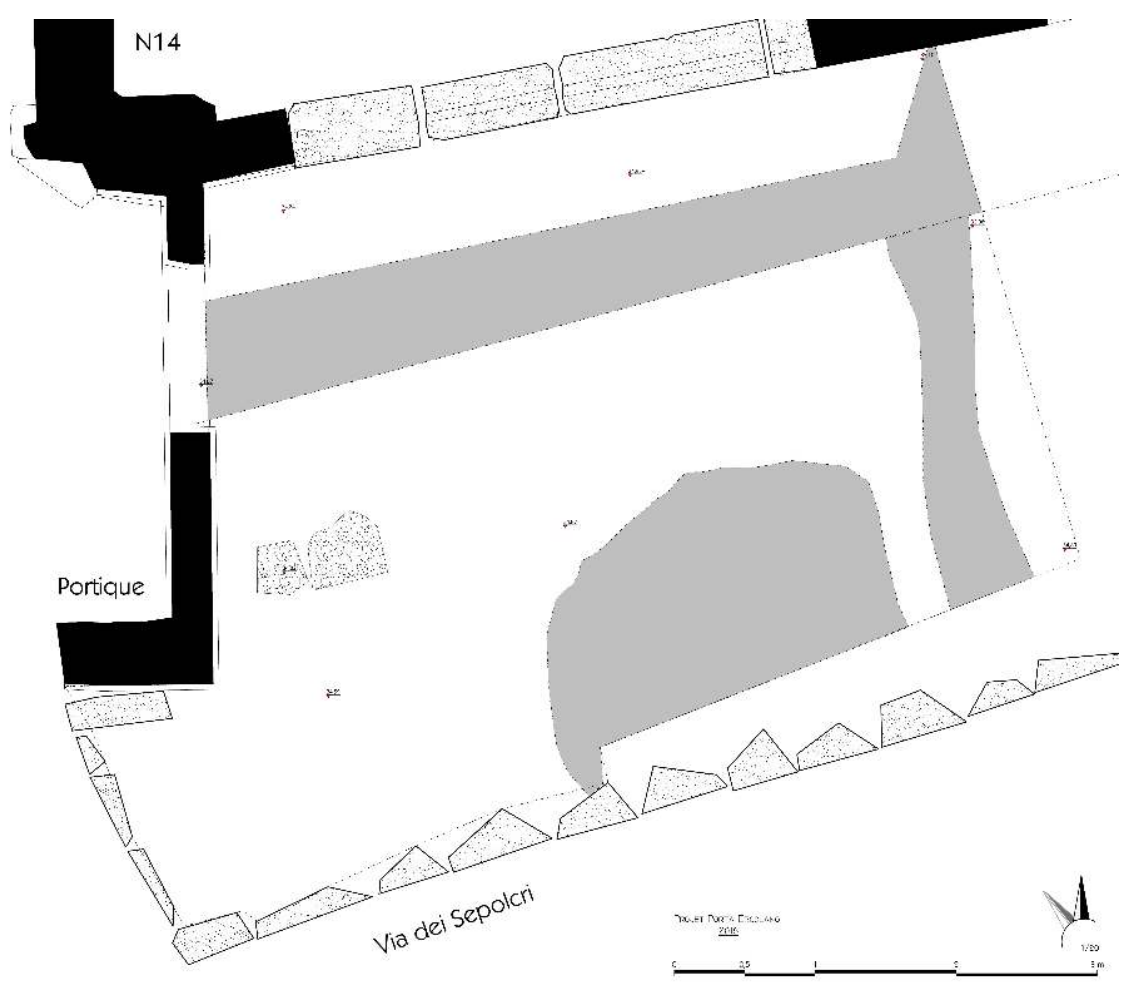

Élaboration S. Zanella.

La première tranchée reconnue, dont les clous de délimitation ont été observés en stratigraphie, a été implantée en correspondance du passage sous l'arche vers l'ouest. Elle court parallèlement aux boutiques 13-14 pour s'achever devant la porta de la villa delle Colonne a mosaico. Une deuxième longe l'intégralité de la limite ouest de notre sondage en croisant à angle droit la première tranchée, devant le piédroit entre N13 et N14. Une troisième, de forme arrondie croise la tranchée précédente vers la limite du trottoir, côté via dei Sepolcri, jusqu'à environ la moitié de notre aire d'investigation. Elle continue très probablement sous la chaussée de la via dei Sepolcri, comme en atteste l'irrégularité des dalles de basalte en ce point. De la même manière, devant la fontaine, à l'extérieur du portique, les premiers niveaux rencontrés ont été touchés par des activités modernes, comme en témoigne le matériel retrouvé.

Dans les deux secteurs, il a été possible d'atteindre les niveaux géologiques sur lesquels ont été réalisées les structures les plus anciennes que nous avons pu observer. Devant la fontaine, une voie dallée dont l'axe diverge sensiblement de l'actuelle via dei Sepolcri, a été retrouvée à environ $0,40 \mathrm{~m}$ sous le niveau du talus qui, depuis la voie actuelle, monte en légère pente jusqu'au niveau de circulation du portique (fig. 10). 
Fig. 10 - Vue zénithale de la voie dallée interceptée entre le portique et la via dei Sepolcri devant la fontaine N20a.

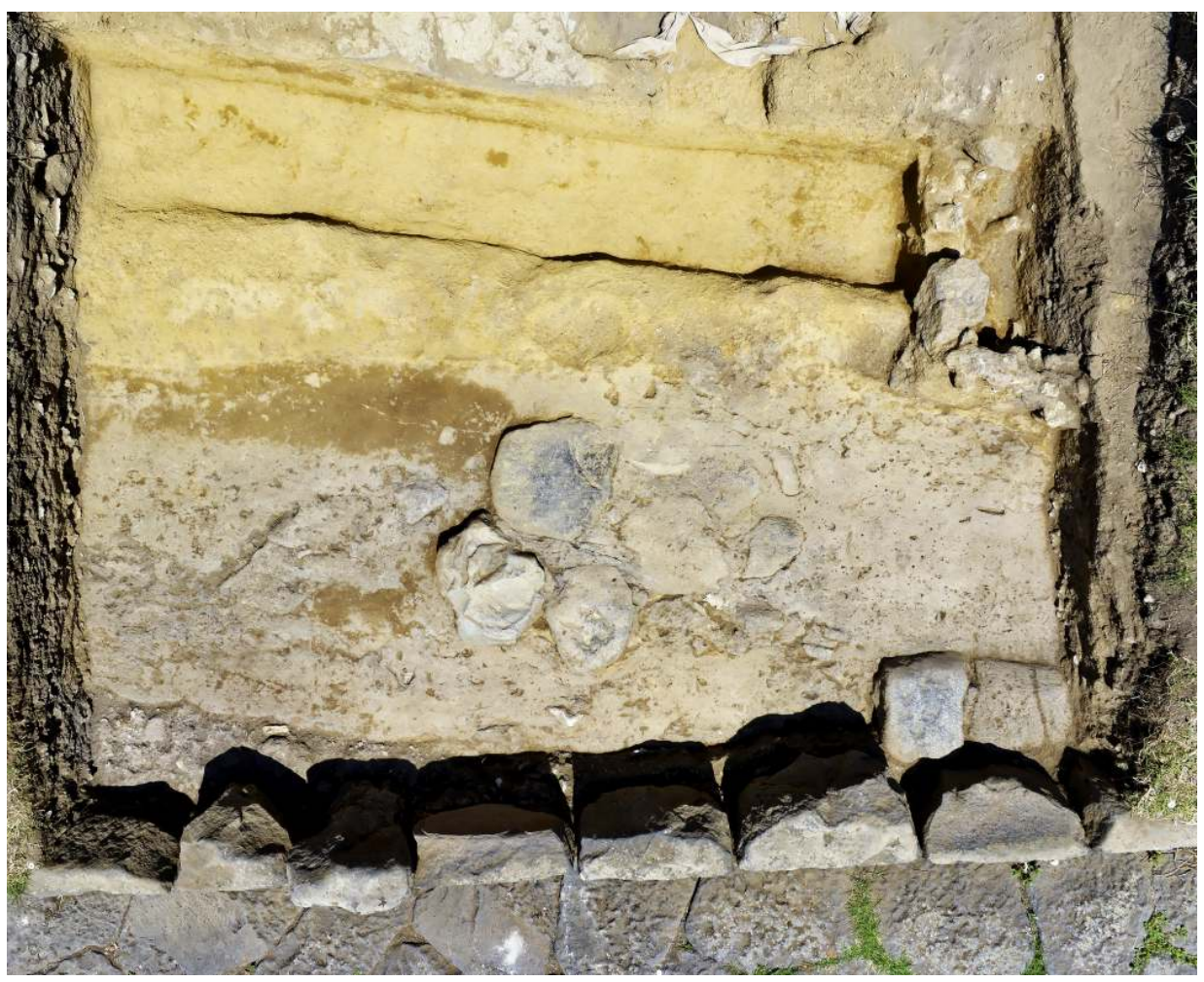

Cliché : B. Lemaire-EFR.

Il est assez probable que cette voie, dont la chaussée porte clairement des marques de recharges et de réfections, soit à mettre en relation avec un segment de voie dallée découvert quelques mètres plus en amont, devant la boutique N14 (fig. 11). Les deux segments sont pavés avec des dalles de basalte de dimensions sensiblement inférieures à celles de la voie en usage en 79. 
Fig. 11 - La voie dallée et les recharges en béton de tuileau interceptées dans le sondage devant la boutique 14, à la base de l'arche est du portique, depuis l'ouest.

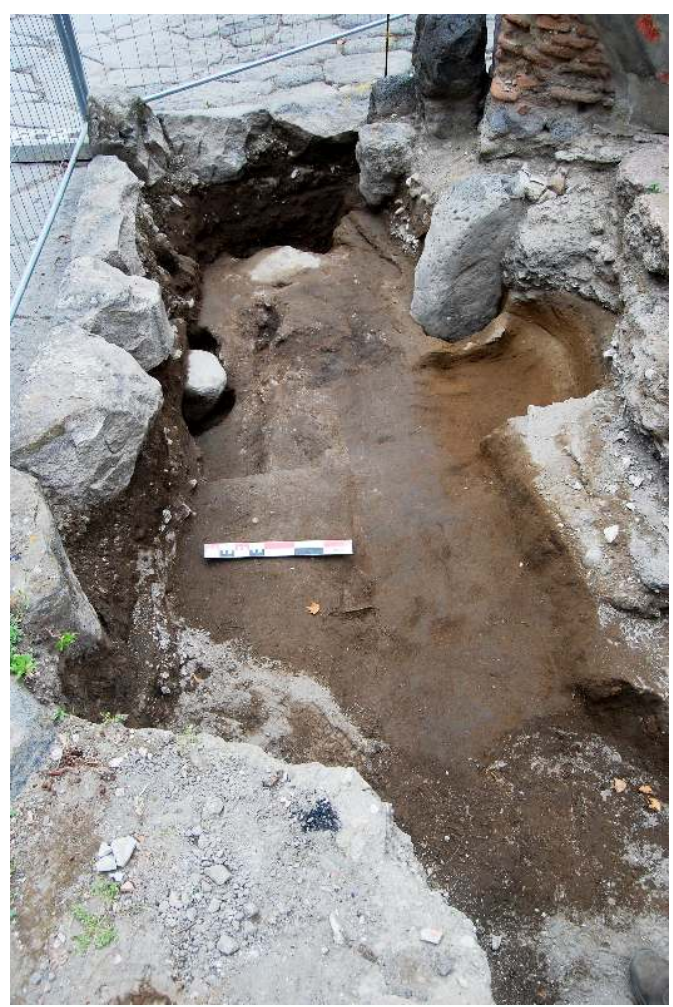

Cliché : A. Delvigne-Ryrko-EFR.

Comme le tronçon devant la fontaine, la chaussée de la voie devant la boutique N14 montre d'importantes recharges, parfois effectuées avec des dalles de béton de tuileau coulé pour niveler les espaces entre les dalles. Bien que les fenêtres dans lesquelles il a été possible d'observer cette voie plus ancienne soient réduites, il semble qu'elle reproduisait le même pendage que celui de l'actuelle via dei Sepolcri. Si, devant la fontaine, la fouille s'est arrêtée sur la voie dallée, devant la boutique N14, nous avons procédé au démontage d'une dalle ainsi que des recharges de tuileau. Cela nous a permis de remettre au jour autre un niveau antérieur de voie, en terre battue, d'une épaisseur d'environ 0,40 m, posée directement sur le niveau géologique bordant la voie (fig. 12). Ce talus présente une pente sensible vers le nord, avec une différence d'environ $1 \mathrm{~m}$ entre le niveau enregistré à la base du portique et devant le seuil de la boutique N14. 
Fig. 12 - Sondage aux pieds de l'arche est du portique. Dernière voie en terre battue en cours de fouille.

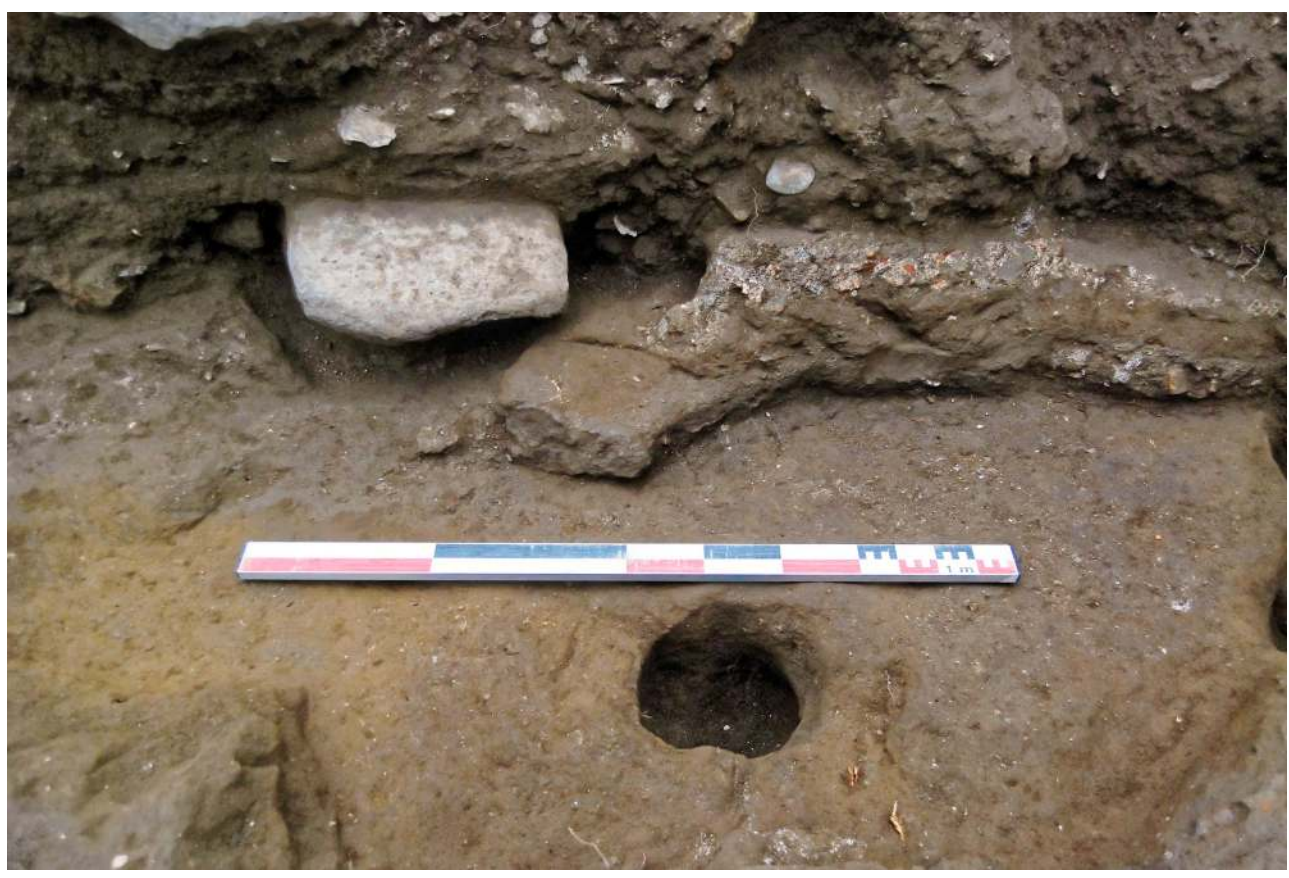

On remarque le niveau géologique sous-jacent et la voie en dalle de basalte qui vient couvrir la première voie.

Cliché : A. Delvigne-Ryrko-EFR.

Bien que nous n'ayons pas pu l'observer sur toute sa continuité, il est très probable que, au niveau de la chaussée, le sol géologique ait été surcreusé lors de l'installation de la première voie en terre battue.

Devant la fontaine, il a été également possible d'intercepter l'ancien trottoir, dont la tranchée de spoliation offre d'importantes données quant à l'orientation de cette ancienne voie (fig. 10). En effet, nous avons pu observer que la chaussée était décalée vers le nord par rapport à la via dei Sepolcri. Une orientation divergente par rapport à cette dernière est également appréciable bien qu'il soit difficile, en raison du caractère réduit de la superficie d'intervention, d'en apprécier davantage l'évolution (fig. 13). La projection idéelle de l'orientation du trottoir et la découverte de la voie dallée aux pieds de l'arche laissent supposer que le tracé de cette voie devait, comme celui de l'actuelle via dei Sepolcri, marquer dans le tronçon oriental un virage en correspondance de l'accès à la villa delle Colonne a mosaico. 
Fig. 13 - Projection idéale du tracé de l'ancienne voie dallée.

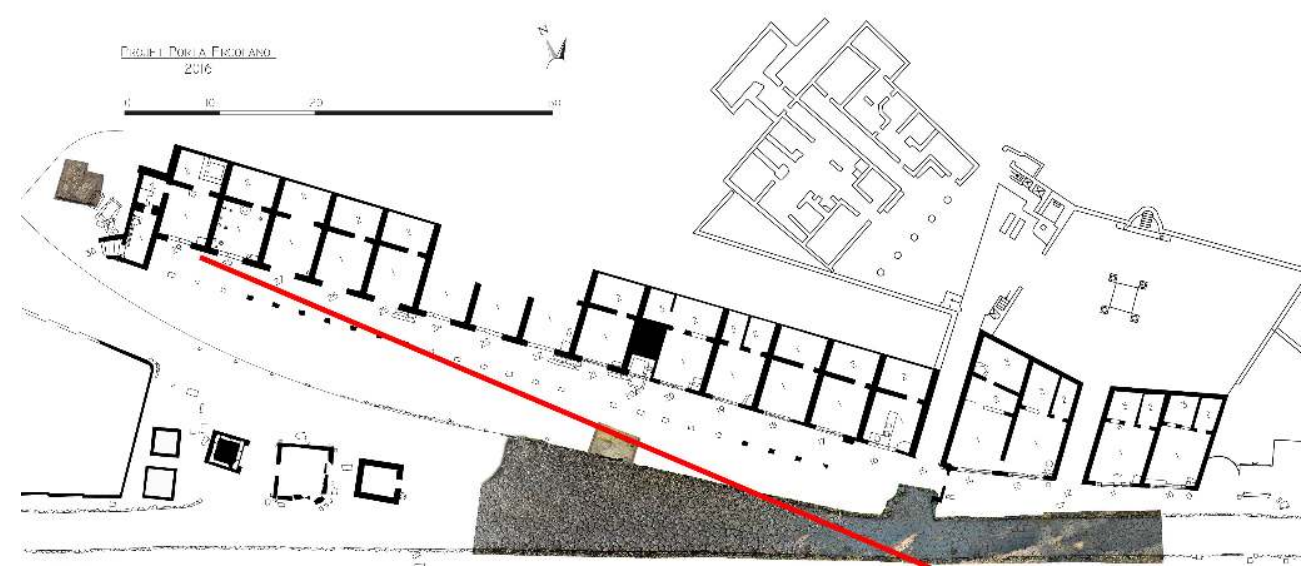

Élaboration S. Zanella-EFR.

17 Il n'est pas à exclure que ce tracé, légèrement divergent, ait été maintenu lors de l'installation du portique. Du moins, telle est la situation que nous pouvons clairement observer devant la boutique N14 où un chasse-roue en basalte, clairement destiné à protéger le piédroit méridional de l'arche d'accès au portique, est installé en perforant les niveaux plus anciens. Sur ces derniers, une autre surface en terre battue, épaisse d'environ 0,10 à $0,15 \mathrm{~m}$ est aménagée ; elle a servi comme niveau de chaussée (fig. 14). L'observation d'une ornière frôlant le chasse-roue confirmerait que l'on est en présence de la voie contemporaine du portique.

Fig. 14 - Aux pieds de l'arche ouest.

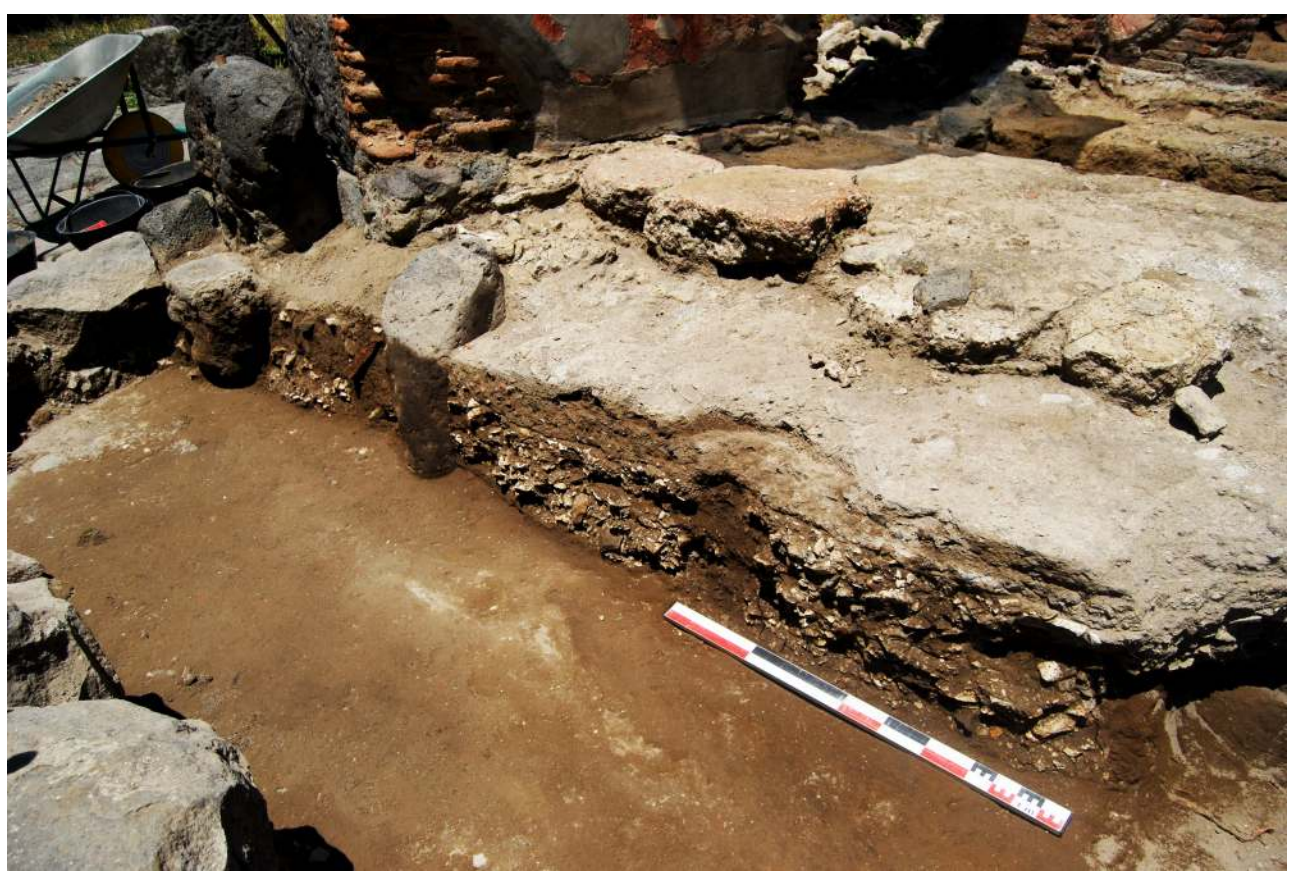

En premier plan la dernière voie en terre battue en usage après la réalisation du portique. Sur la droite de la photographie, le chasse-roue en basalte pris dans les niveaux successifs et l'ornière qui vient le frôler.

Cliché : S. Zanella-EFR 
la fontaine, en revanche, nous n'avons pas pu observer de niveaux équivalents, très probablement en raison des travaux engagés lors de l'installation de l'actuelle via dei Sepolcri.
L'installation du portique a dû entrainer des travaux d'envergure comme l'attestent les installations de levage, interceptées en correspondance des piédroits des arcades du portique devant la fontaine (fig. 15).

Fig. 15 - Portique devant la fontaine N20a. Aménagements en lien avec la mise en place de machine pour l'élevage des blocs des arcades du portique.

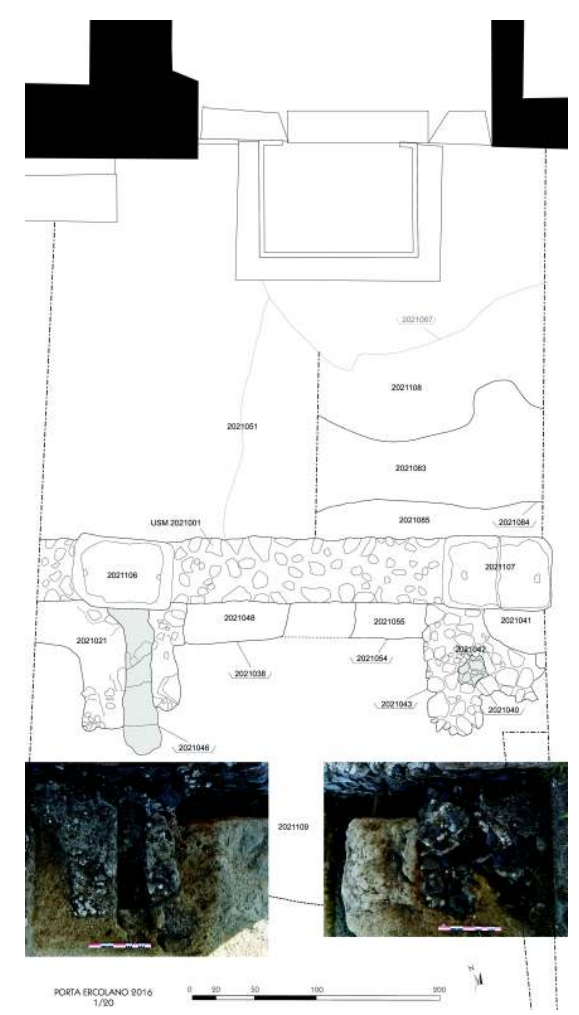

B. Lemaire / S. Mencarelli / S. Zanella-EFR.

Ces installations, qui se traduisent par l'aménagement d'importants empierrements autour de cavités, sont réalisées directement sur le substrat dans lequel sont également érigées les boutiques. Les machines de levage sont installées peu après la réalisation de la fondation continue du portique. Déjà observé lors des précédentes campagnes en avant de la boutique $\mathrm{N}^{2} 9^{3}$, ce massif est réalisé, comme le ressaut de fondation, en opus incertum de lave. Des niveaux de circulation postérieurs à l'installation du portique ont pu être mis en évidence à la fois devant la fontaine et devant la boutique N14. Dans les deux cas, il s'agit de niveaux en terre battue très indurés; devant la boutique N14, un niveau chargé en galets recouverts par un béton de tuileau a été également mis au jour. Malheureusement, il est impossible d'établir un lien avec les niveaux plus anciens de la voie en raison de la tranchée moderne qui a coupé la stratigraphie entre les parties septentrionale et méridionale de la zone de fouille. Ces niveaux sont antérieurs à la mise en place du seuil de la boutique N14 : ils ont été creusés par la tranchée d'installation de celui-ci.

Devant la boutique N14, avant l'installation de la via dei Sepolcri, un pendage assez important existait entre le trottoir devant de la boutique et la voie en contrebas. Après 
une première phase dans laquelle cette situation est maintenue, même après l'érection du portique, des travaux de nivellement sont réalisés. Ceux-ci ont été plus particulièrement observés entre le chasse-roue aux pieds de l'arche et l'actuelle via dei Sepolcri. En ce point, en effet, la recharge, installée pour rattraper la pente, présente une épaisseur plus importante, ce qui en a permis une conservation optimale. Au sommet, devant la boutique N14 et l'arche, les travaux modernes et les eaux de pluie ont profondément érodé la stratigraphie, en compromettant le lien entre les deux secteurs.

Nonobstant cette perte, au moins trois recharges de la dernière voie en terre battue (en phase avec la réalisation du portique) sont attestées avant la construction de l'actuelle via dei Sepolcri. Ces niveaux, mis en place pour niveler le secteur, atteignent une épaisseur d'environ $0,60 \mathrm{~m}$ au niveau du piédroit méridional de l'arche et présentent un pendage doux vers les blocs de délimitation du trottoir actuel (fig. 14).

Sur ces recharges, ainsi que sur les niveaux de trottoir plus anciens, entre la tranchée moderne et le chasse-roue, deux séries de trois trous de poteaux ont été observées. La séquence stratigraphique confirme que ces aménagements sont en lien avec les travaux de réfection de l'arche, dont les éléments en grand appareil ont été remplacés par un parement en fragments de tuiles. Les niveaux mis en place pour adoucir la pente entre le seuil de la boutique N14 et l'actuelle via dei Sepolcri, sont par ailleurs percés par la tranchée de fondation du trottoir de la voie dallée. Dans l'état actuel, il est donc vraisemblable que nous nous trouvions là face à une phase de profond réaménagement du secteur quand, en même temps que la réfection de l'arche, la via dei Sepolcri fut réalisée avec un nouveau tracé et une chaussée de basalte. Un dernier niveau de sol, dont seules deux plaques de béton de tuileau sont encore conservées à la base du piédroit méridional de l'arche, a dû être installé sur l'intégralité du trottoir.

Devant la fontaine, des travaux étaient en revanche encore en cours au moment de l'éruption. Les fouilles ont en effet pu intercepter une fosse profonde qui, partant de la base de la fontaine et passant sous les fondations du portique, se terminait en équerre au sud. L'axe de la fosse est centré entre les deux piliers de l'arcade (fig. 16). La fosse perce les niveaux géologiques jusqu'à environ $2,40 \mathrm{~m}$ de profondeur ; elle était remplie de lapilli non remaniés lors de la fouille. 
Fig. 16 - Portique devant la fontaine N20a. La fosse antique et les niveaux plus récentes interceptés dans le secteur.

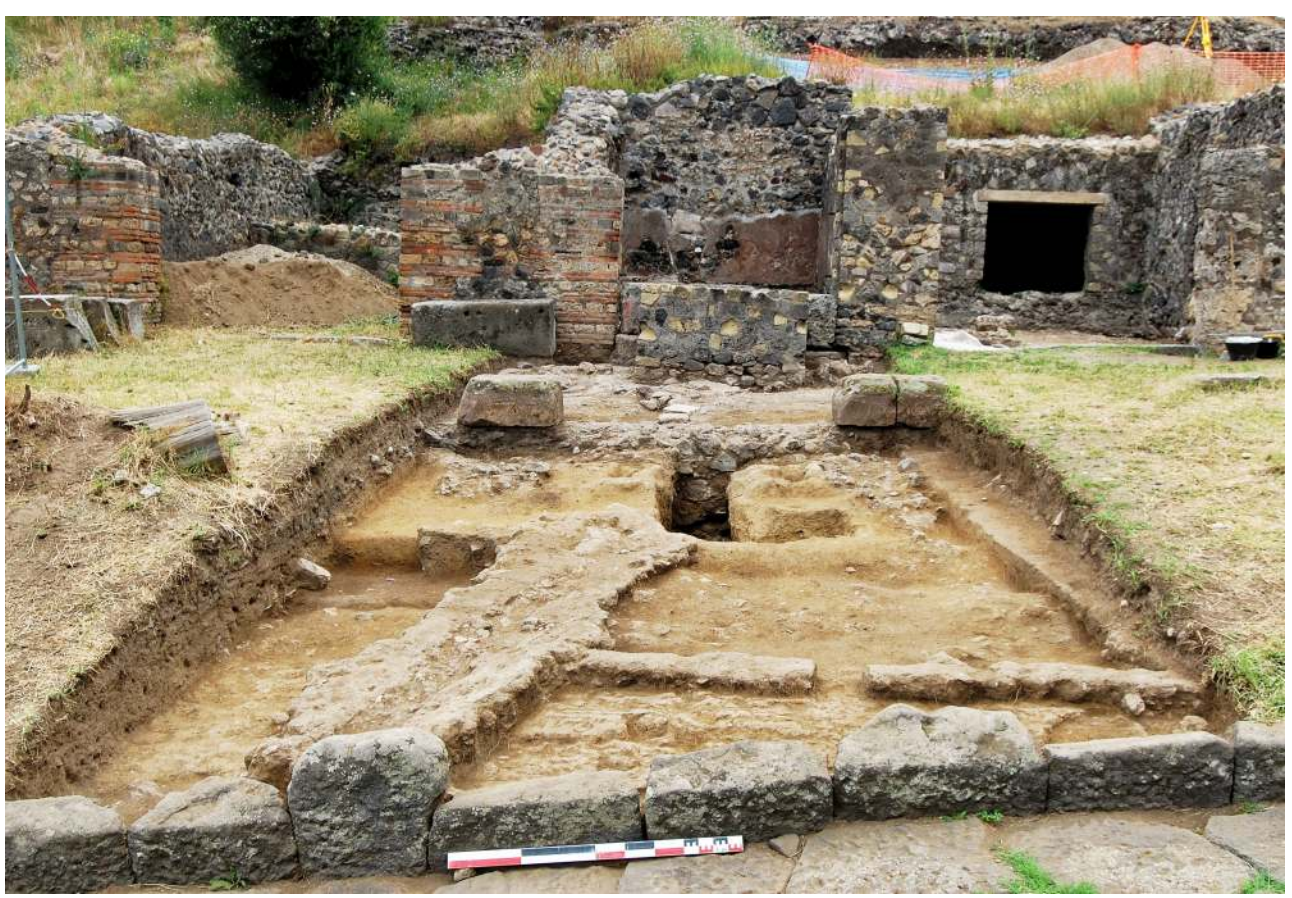

Vue depuis la via dei Sepolcri.

Cliché : B. Lemaire-EFR.

En synthèse, les sondages pratiqués sous le portique et entre celui-ci la via dei Sepolcri ont permis de retracer un niveau de voie dallée précédant le tracé actuel, ainsi que ses différentes recharges. Cette artère, dont l'étude du matériel permettra de préciser la chronologie, diverge par rapport à la voie actuelle avec un parcours déplacé vers le nord. Devant la fontaine, un alignement différent a pu être également observé. Ce parcours est encore en usage lors de la réalisation du portique. Le chasse-roue au pied de l'arche devant la boutique N14 confirme la coexistence d'un tracé initial sensiblement plus au nord et de l'arche elle-même. Dans un deuxième temps, d'importantes recharges ont progressivement nivelé la pente entre le trottoir en avant de la boutique N14 et l'ancienne voie, jusqu'à ce que la mise en place de l'actuelle via dei Sepolcri parachève ces transformations.

\section{La boutique N14 : un espace à peine achevé}

25 À la suite des travaux effectués en 2015, la boutique N14 a été de nouveau fouillée cette année, en se concentrant exclusivement sur la pièce N14/1. La première phase observée correspond à un silo, déjà rencontré lors de la campagne précédente dans la pièce N14/2. Directement creusé dans le substrat éruptif, la majeure partie de ce silo devait d'étendre dans la partie occidentale de la boutique N13 voisine (fig. 17). Le fait le plus frappant reste la proximité du sommet de la coupole de ce silo avec le sol en fonction lors de l'éruption du Vésuve. 
Fig. 17 - Boutique N14. Vue du silo après la fouille de son comblement d'abandon.

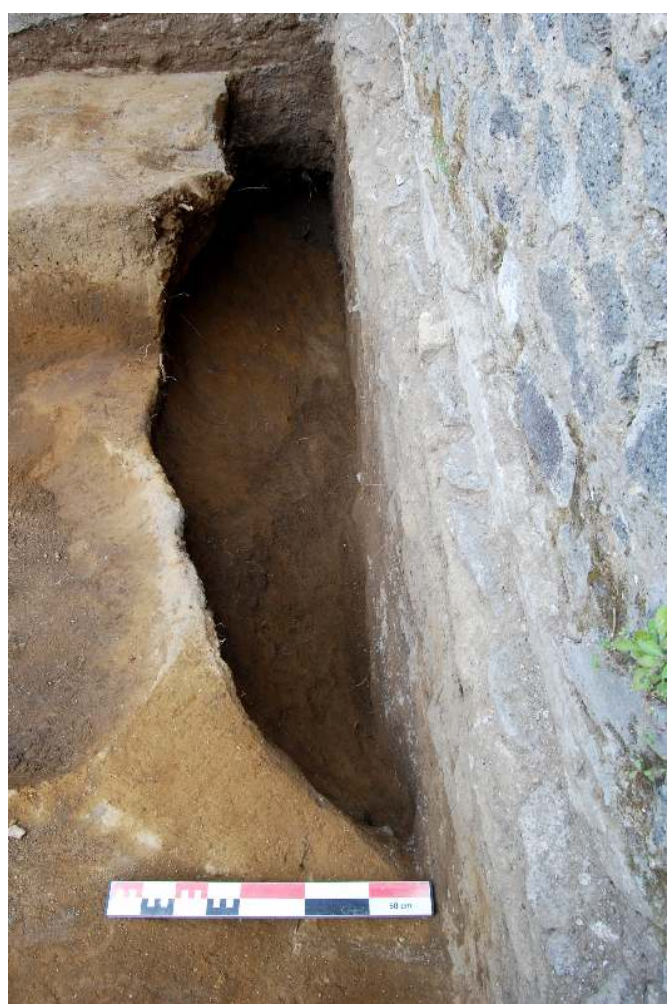

Vue du sud

Cliché : A. Delvigne-Ryrko-EFR.

Bien qu'il soit évidemment impossible de déterminer la hauteur du conduit qui permettait d'accéder à ce silo ou l'épaisseur de terre nécessaire pour éviter son effondrement en cas de circulation à sa surface, sa situation par rapport au sol de la boutique est l'argument qui permet le mieux de mettre en évidence les travaux de terrassement menés à une période inconnue dans ce secteur ${ }^{4}$.

La phase suivante, antérieure à la construction des boutiques N10-13, est marquée par le creusement d'une série de fosses dont seule la plus récente autorise une interprétation. Elle est constituée d'une juxtaposition d'au moins trois fosses aménagées les unes contre les autres dans un espace de forme vaguement trapézoïdale s'achevant dans la boutique N13. Le fond de deux de ces fosses, situées au nord et à l'est de l'ensemble, tend vers l'horizontalité, à une altitude comparable. Elles sont séparées par une fine épaisseur de substrat. La fosse la plus imposante se développe dans l'angle sud-ouest de l'ensemble (fig. 18-19). 
Fig. 18 - Boutique N14. Relevé photogrammétrique en plan de l'ensemble de la boutique.

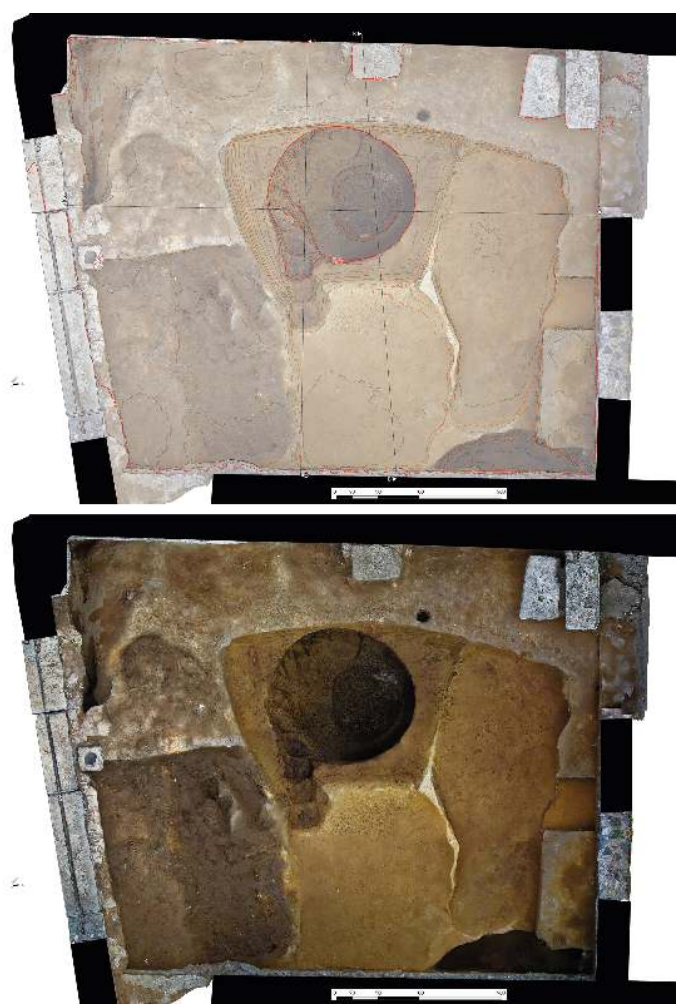

En haut, indication des lignes de coupes et des isoplèthes d'altitude (chaque 0,05 m) Échelle : 1/20.

Photogrammétrie : S. Zanella / N. Monteix-EFR. 
Fig. 19 - Boutique N14. Coupes photogrammétriques B-B', C-C' et D-D' du système de fosses.
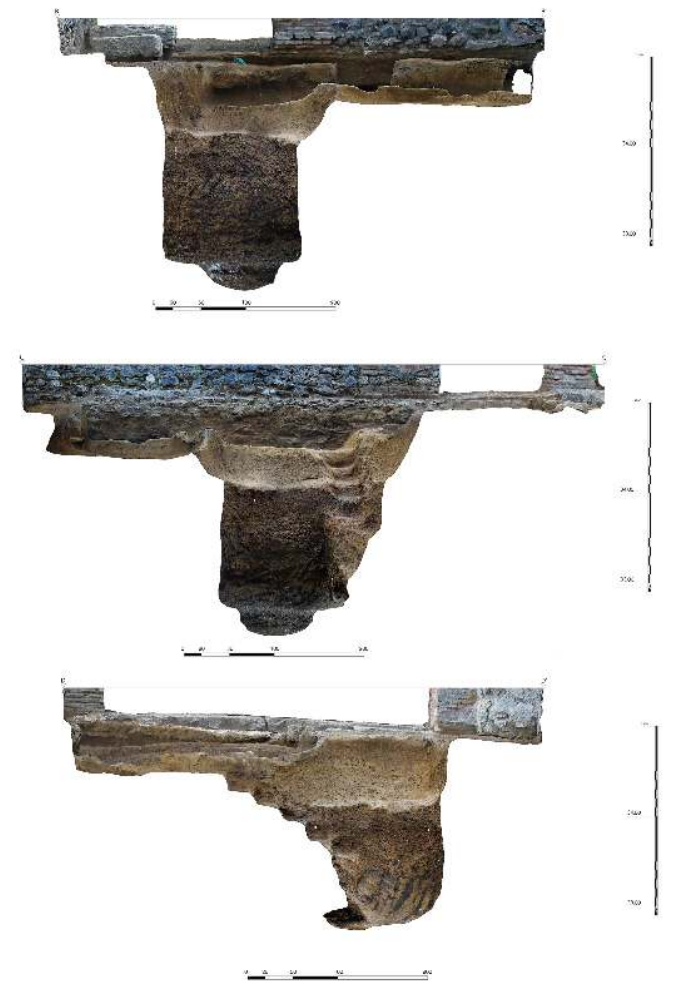

Échelle : 1/20.

Photogrammétrie : S. Zanella / N. Monteix-EFR.

À son sommet, elle présente un plan sub-trapézoïdal avec des parois faiblement inclinées avant de former un palier. Là, la fosse adopte un plan circulaire avec un diamètre de $1,80 \mathrm{~m}$ tandis que ses parois deviennent verticales sur une profondeur de $1,45 \mathrm{~m}$. Le creusement principal, effectué à travers la stratigraphie éruptive antéplinienne - remaniée par colluvionnement dans sa partie supérieure?--, s'achève sur le plan supérieur de la coulée de lave de l'éruption du Mercato. Celui-ci est creusé sur un diamètre de $0,90 \mathrm{~m}$ et une profondeur de $0,26 \mathrm{~m}$. Pour accéder à cette profondeur, un escalier en colimaçon a été sculpté dans la paroi de la fosse. Cinq marches sont parfaitement conservées, tandis que les autres ont été trouvées par blocs assez importants dans le comblement de la fosse. Des traces de pics conservées dans la paroi attestent de leur destruction. Pour démesuré qu'il puisse paraître, cet aménagement doit être interprété comme une tentative d'atteindre la lave sous-jacente dans l'espoir de l'exploiter. Sa nature extrêmement friable a fait que cette tentative a finalement avorté et que - de dépit? - ses promoteurs ont remblayé les différentes fosses rapidement et sans ménagement. On notera cependant que le substrat volcanique supérieur, ce sable jaune rapidement incohérent a toutefois été exploité, vraisemblablement pour confectionner du mortier.

La phase suivante, a priori immédiatement postérieure, a vu l'érection des différentes boutiques N10 à N14. Dans le cas de N14, cette construction était, selon toute probabilité, encore en voie d'achèvement au moment de l'éruption du Vésuve. 


\section{La boutique N20 : un atelier de production polymétallique?}

La boutique N20 est actuellement organisée en trois pièces disposées en équerre, entourant la pièce aveugle servant de probable réservoir à la fontaine N20a. Le choix d'intervenir dans ces espaces a été initialement motivé par la possibilité théorique de bénéficier, dans les deux arrière-boutiques, d'une accumulation stratigraphique liée au besoin de récupérer la pente, et dont l'observation aurait permis de mieux cerner les évolutions de la file de boutiques situées au nord de la via dei Sepolcri. Il s'agissait, en outre, de définir les fonctions commerciales ou de production implantées dans ces locaux non interprétés jusqu'alors. Par ailleurs, il convient de noter que, bien que bénéficiant de bonnes conditions globales de conservation, la boutique et ses dépendances ont subi au fil de leur histoire, de forts remaniements qui ont souvent impliqué l'arasement des éléments antérieurs, en particulier des niveaux de sol. De ce fait, la présentation qui suit des différentes phases reste préliminaire.

Aucune occupation antérieure à l'installation de la boutique n'a été observée : tous les murs périmétriques sont érigés au cours d'une unique phase - comportant plusieurs moments de chantier - implantée directement sur des niveaux éruptifs anté-pliniens éventuellement remaniés par colluvionnement. La boutique dispose alors de deux pièces, N20/1 en façade dont le plan est resté globalement inchangé malgré les diverses transformations successives, et N20/2-3, une arrière-boutique barlongue qui fut par la suite subdivisée en deux espaces. L'étage, planchéié, était accessible depuis le portique par une entrée indépendante donnant sur un escalier dont seule la base était maçonnée. Il est impossible de déterminer les premières activités développées dans la boutique : seuls les maigres vestiges d'un niveau de béton de tuileau ont été observés dans la pièce N20/1. On supposer que, dans l'angle nord-oriental de N20/2-3, une citerne a alors été installée. Son conduit de puisage, creusé dans le substrat, n'est pas maçonné, cependant, sa paroi est surcreusée à intervalles réguliers d'échelons permettant d'y descendre (fig. 20), a eu lieu lors de cette phase. Aucun niveau de sol en usage lors de cette phase n'a été conservé dans l'arrière-boutique. 
Fig. 20 - Boutique N20. Vestiges de la citerne située au nord-est de la pièce N20/2-3.

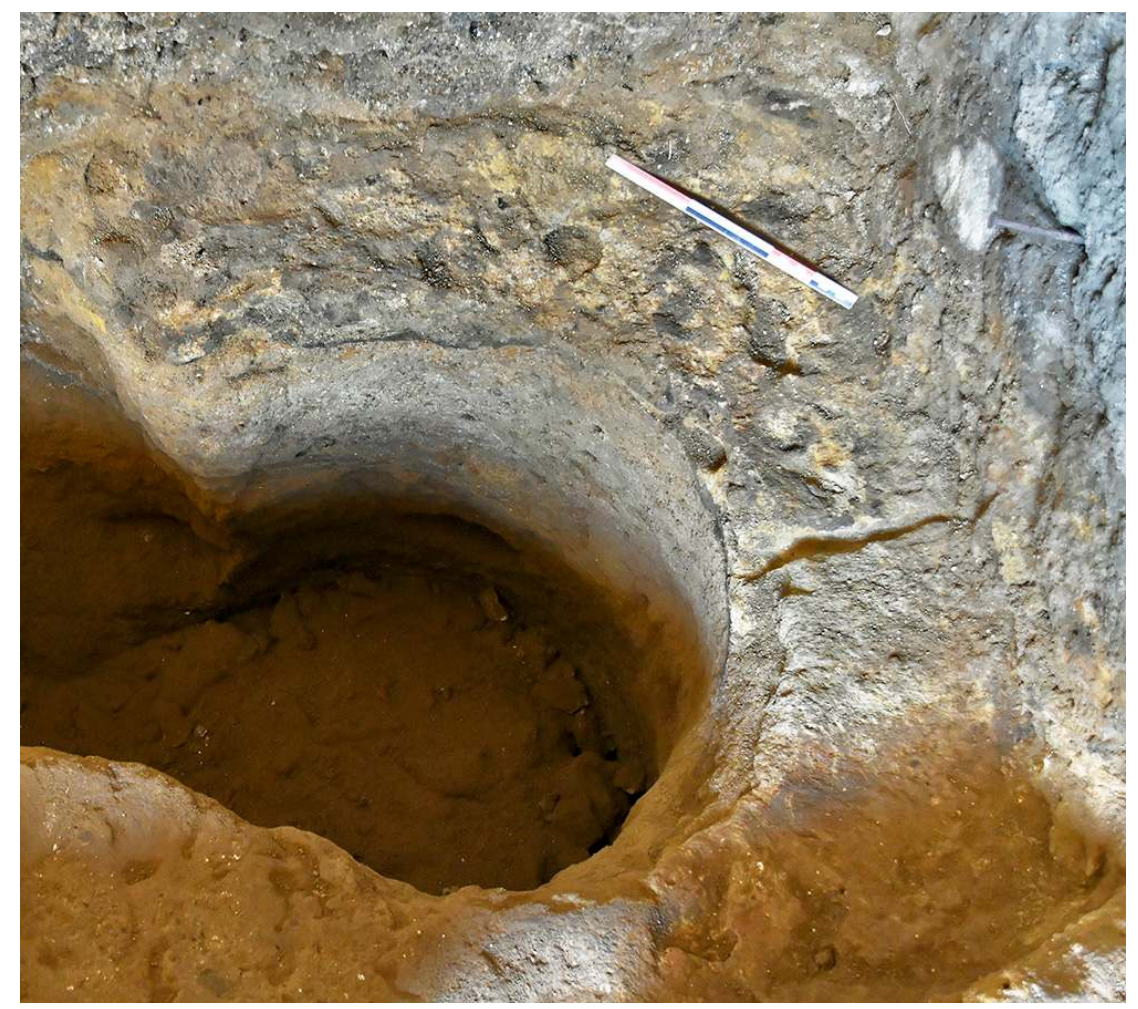

On note un des échelons dans la paroi septentrionale.

Vue du sud.

Cliché : N. Monteix-EFR.

Ce n'est qu'au cours de la phase suivante que d'importants remaniements sont exécutés à la fois dans la pièce principale et dans l'arrière-boutique. La boutique est alors dotée d'un sol en béton de tuileau recouvrant vraisemblablement l'ensemble de la surface, dans lequel sont incorporés un bassin contre le mur oriental ainsi qu'une citerne, suite au comblement de la citerne précédemment située dans la pièce N20/2-3. Un parcours d'évacuation de l'eau est ménagé entre la citerne et l'angle sud-occidental de la pièce, où un avaloir traverse le mur pour vraisemblablement rejoindre le trop-plein de la fontaine N20a (fig. 21). Ce déplacement de la citerne pourrait être lié à la mise en place d'une activité de production de nature indéterminée dans les différentes pièces de la boutique. 
Fig. 21 - Boutique N20. Aménagements hydrauliques installés au cours de la seconde phase.

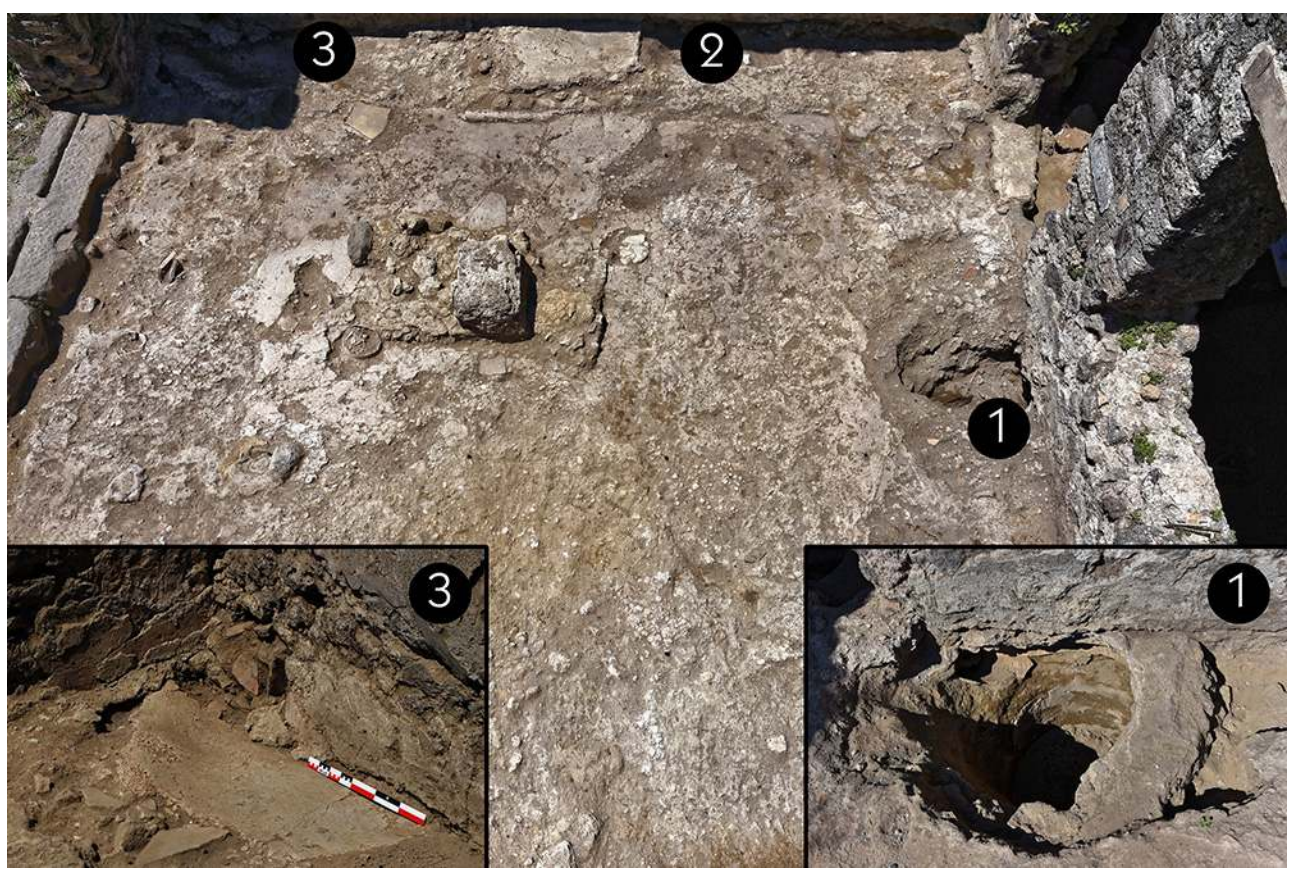

1. Citerne au conduit maçonné. 2. Bassin quadrangulaire. 3. Avaloir traversant le mur occidental. Vue générale de la boutique prise de l'est.

Clichés : N. Monteix / S. Zanella / A. Delvigne-Ryrko-EFR.

Pendant la troisième phase, d'importants travaux structurels et fonctionnels sont menés. Le mur de refend entre les pièces N20/1 et N20/2-3 est abattu. Un nouveau refend est construit de manière à ce qu'il puisse recevoir une voûte qui se développe en longueur au-dessus de la pièce N20/2-3. Le principal aménagement est constitué par une fosse de forme sub-circulaire en plan, d'un diamètre d'environ $0,90 \mathrm{~m}$ dans son axe longitudinal et d'environ $0,77 \mathrm{~m}$ dans son axe transversal. Cette fosse se développait sur une hauteur d'au moins $1,32 \mathrm{~m}$. Ses parois verticales ont reçu un cuvelage peu épais, dont le parement interne n'est que très grossièrement lissé. Au sommet de la partie cylindrique, un évasement, aménagé avec des fragments d'amphores vinaires, est conservé. On pouvait accéder à cette fosse par un escalier disposé suivant un axe sud-est/nord-ouest, composé à l'origine de cinq marches. Bien qu'une tranchée postérieure à l'éruption ait emporté une partie de cette construction, une interprétation raisonnable que l'on peut proposer est celle d'une fosse de coulée pour des alliages cuivreux dans laquelle les moules des objets à fondre étaient cuits et décirés, puis, laissés en place, ils recevaient le métal en fusion $^{5}$ (fig. 22). 
Fig. 22 - Boutique N20. Coupe et relevé en plan photogrammétriques de la probable fosse de coulée.
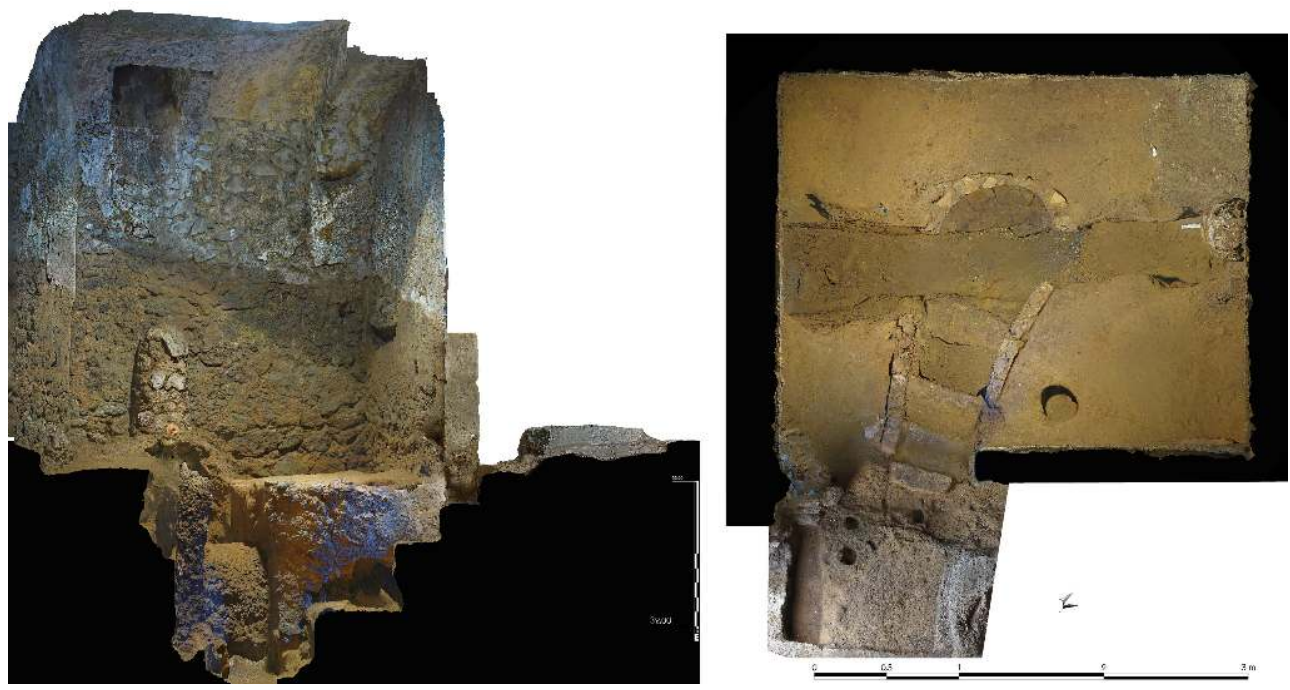

Échelle : 1/20.

Photogrammétrie : S. Zanella / N. Monteix-EFR.

Malgré l'absence de restes de coulées ou de fragments de moules - mais les sols ont été détruits et les déchets vraisemblablement jetés lors du comblement de la seconde citerne -, d'un point de vue morphométrique, le parallèle entre cette fosse et les autres attestations connues est frappant. Un autre aménagement pourrait avoir servi dans le cadre de cette production. Ainsi, une amphore vinaire locale a été insérée dans le sol à l'horizontale (fig. 23). Des traces de chauffe à proximité ainsi que la situation de cette amphore en remploi permettent de pointer vers une utilisation comme four de recuit. 
Fig. 23 - Boutique N20. Amphore en remploi dans le sol de la pièce N20/2-3, éventuellement utilisée comme four de recuit.

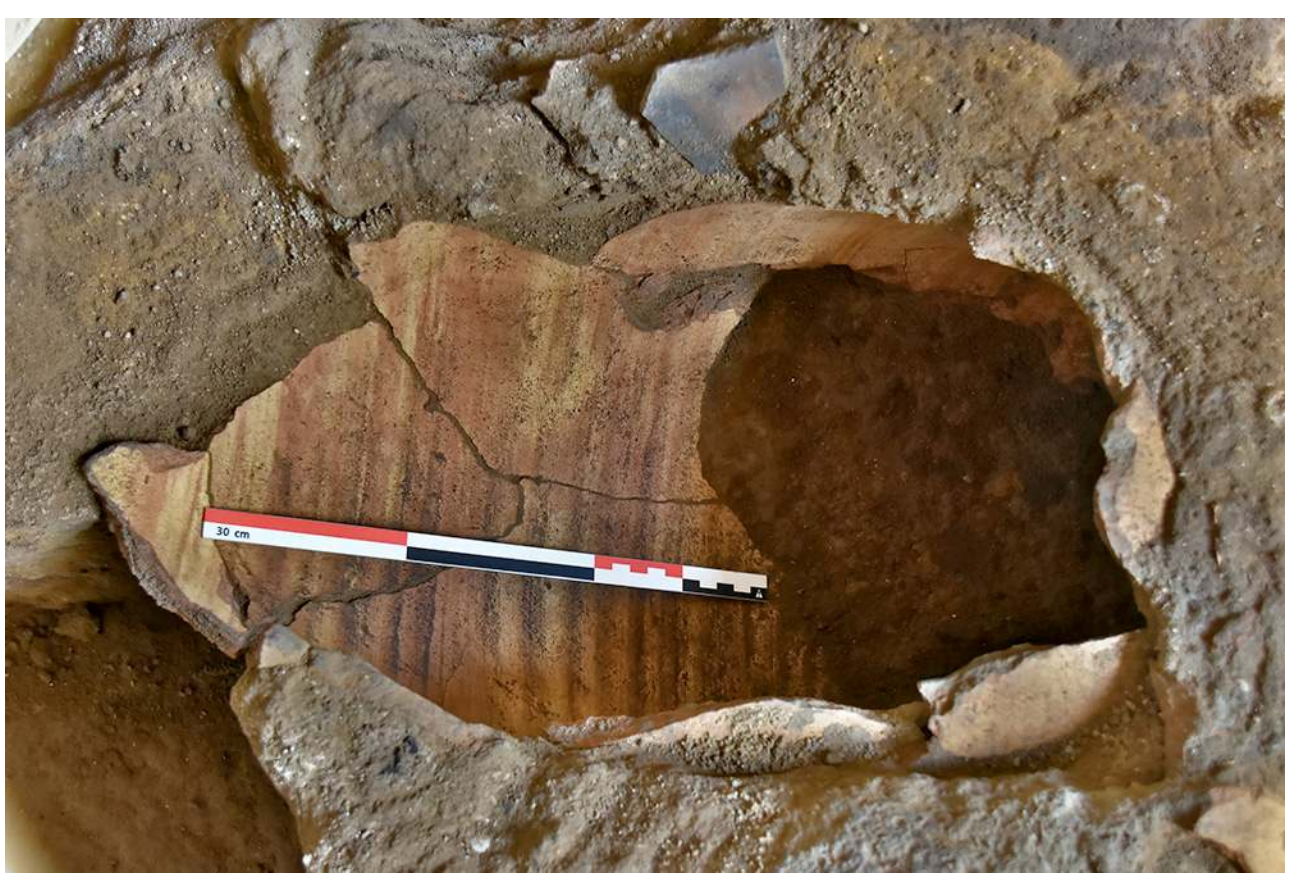

Vue de l'ouest.

Cliché : N. Monteix-EFR.

Dans la pièce de façade, des aménagements liés à cette nouvelle activité sont construits. Au centre de la pièce, un bloc de maçonnerie est installé directement sur le sol en béton de tuileau (fig. 24). Si sa construction est de piètre qualité, il est renforcé en son centre par un bloc plus imposant en calcaire «du Sarno». Son angle sud-oriental comprend, fiché dans le sol, un fond de vase. Autour de ce massif, quatre cavités de faible profondeur ont été ménagées. 
Fig. 24 - Boutique N20. Massif maçonné et aménagements périphériques, possibles traces d'un atelier de métallurgie du fer.

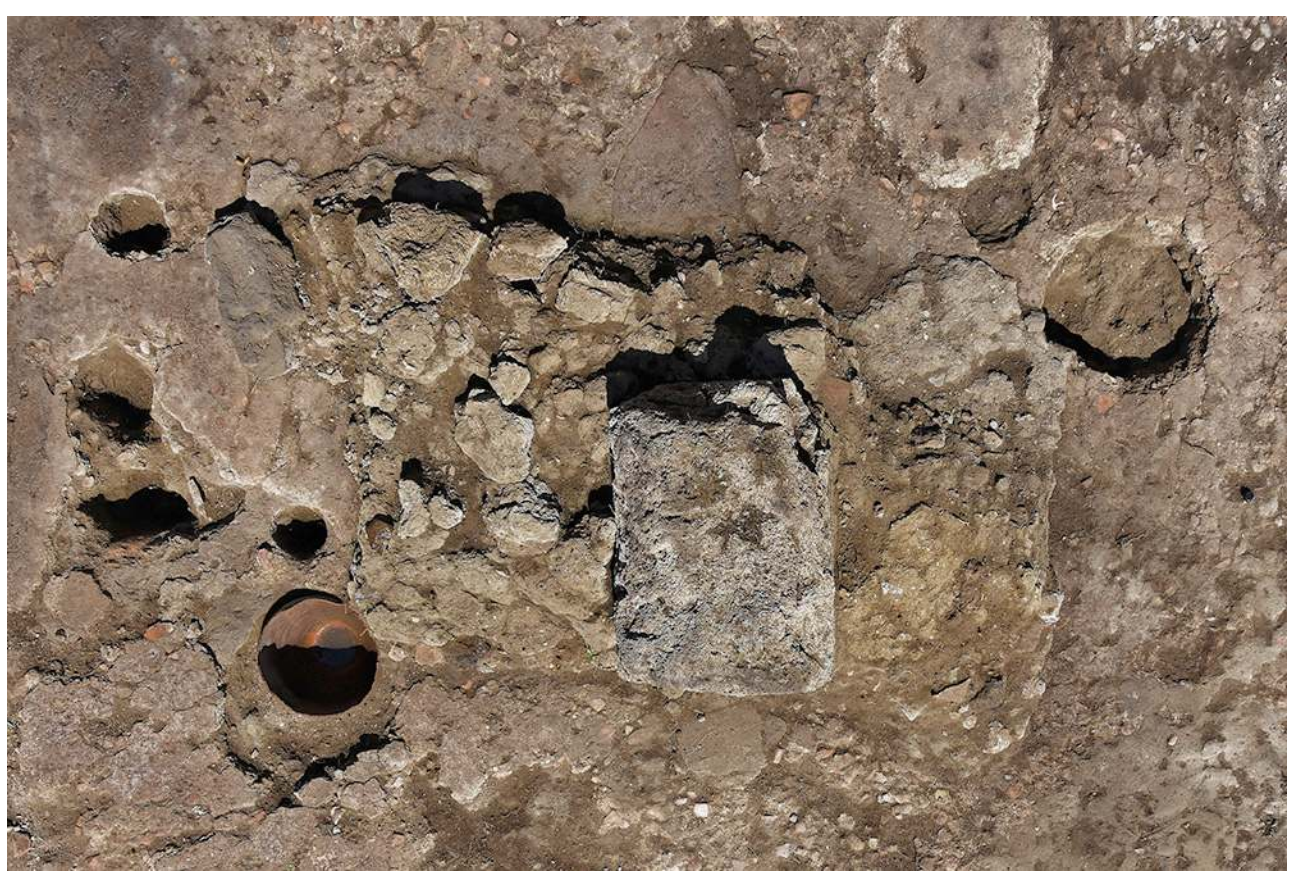

Vue zénithale de l'est.

Cliché : N. Monteix-EFR. de fer, ainsi que la découverte, dans le comblement de la citerne survenu ultérieurement, de trois scories de fer en calotte, correspondant normalement au résultat d'une journée de travail, pointent vers l'installation d'un espace de métallurgie du fer. Avec toute la prudence qu'impose la relative rareté des traces indubitables de travail des alliages cuivreux et du fer - en cours d'étude ${ }^{6}$-, il apparaît possible de proposer que, lors de cette phase, un atelier orienté vers une production polymétallique a été installé. Pour l'heure, on se contentera de suggérer que le massif maçonné pourrait avoir servi de forge sur table.

La dernière phase, restée inachevée à cause de l'éruption, est caractérisée par un réaménagement massif de l'ensemble des pièces, visant à un changement aussi probable que radical d'activité. Dans l'angle nord-oriental de la pièce N20/2-3, une grande fosse circulaire est creusée, vraisemblablement destinée à permettre de récupérer des matériaux de construction. Une fois son rôle terminé, cette fosse, comme la citerne située dans la pièce N20/1, a été comblée avec les différents éléments détruits par les travaux. La fosse de coulée est également comblée, sans qu'elle n'ait été préalablement vidée des restes carbonisés de sa dernière utilisation ${ }^{7}$. Un mur de refend est érigé pour créer deux arrière-boutiques. Dans la nouvelle pièce N20/3, un pilier maçonné est construit pour servir de protection à un tuyau de plomb, probablement destiné à alimenter en eau sous pression la fontaine N20a. Dans la pièce N20/1, un remblai a commencé à être disposé sur l'ensemble de la surface, après l'arasement de l'éventuelle margelle de la citerne, ainsi que du bassin. L'étage, auparavant accessible depuis le portique, peut désormais être rejoint exclusivement depuis l'intérieur du local. Ces travaux étaient encore inachevés au 
moment de l'éruption, comme en témoigne le tas de chaux disposé contre le nouveau mur occidental de la pièce N20/2 (fig. 25).

Fig. 25 - Boutique N20. Tas de chaux et empreintes de bois pris dans le matériel éruptif.

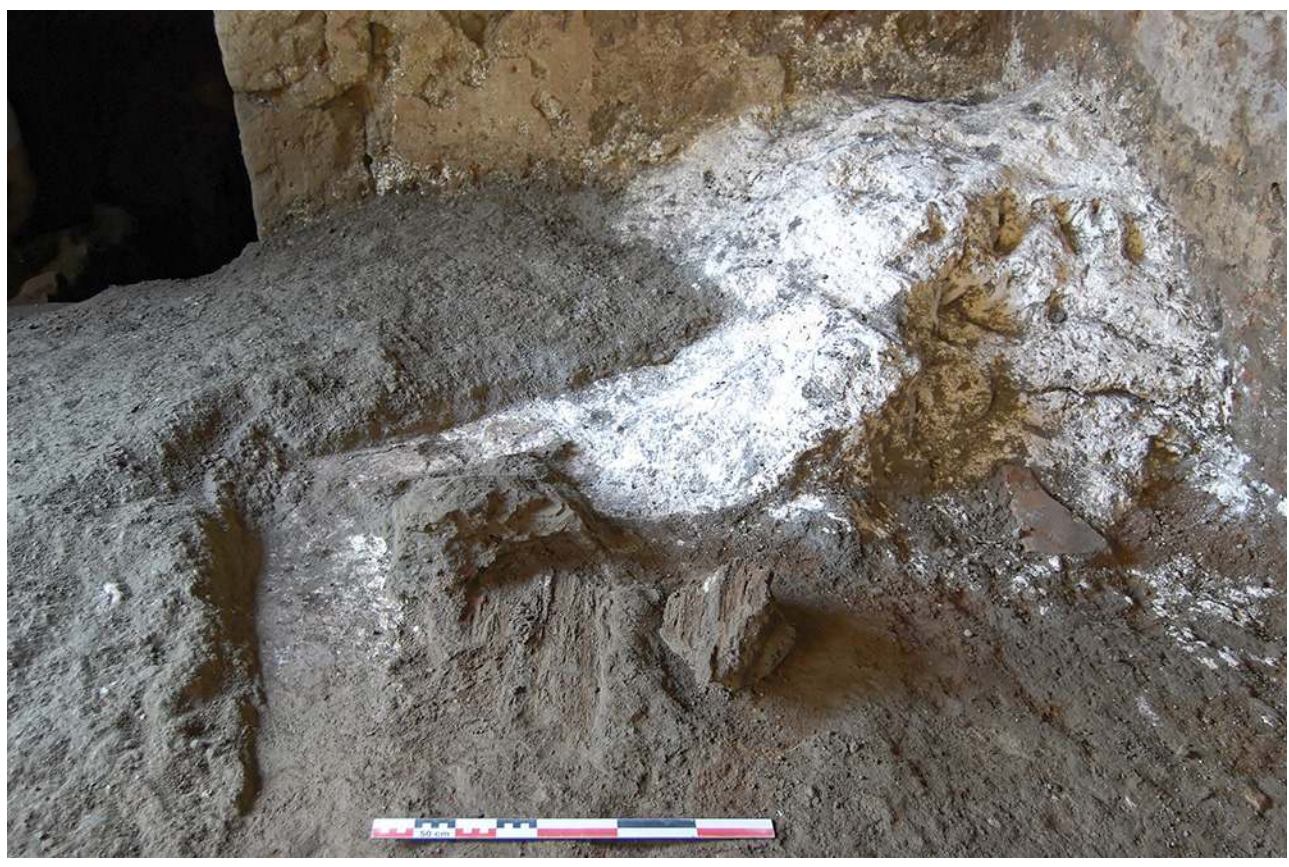

Vue de l'est.

Cliché : A. Denysiak-EFR

Enfin, la fouille a pu mettre en évidence plusieurs passages de pillards à des dates indéterminées mais cependant postérieures, même de peu, à l'éruption. Au moins deux tunnels ont été percés, vraisemblablement l'un à la suite de l'autre. Il semble que le premier tunnel ait été percé dans la pièce N20/3, avec pour objectif principal la récupération du tuyau en plomb qui traversait cet espace : la perforation créée dans la voûte a ensuite été restaurée comme une fenêtre. Les explorateurs/pillards ont traversé la fosse de coulée et ont poursuivi leurs investigations en passant sous le mur sud de la pièce, pour déboucher dans la pièce aveugle au nord de la fontaine N20a. Dans la pièce N20/2, le mur occidental est percé d'un creusement ovale clairement identifiable grâce aux restaurations modernes. Il en va de même pour le mur oriental. Les explorateurs/ pillards sont entrés dans la pièce N20/2 par l'est. Ils ont commencé par dégager la salle d'une partie de son matériel éruptif, pour l'essentiel dans sa moitié occidentale, avant de continuer leur progression vers l'ouest en longeant le mur septentrional. Au cours de ce déplacement, ils ont rencontré le groupe de victimes de l'éruption et, après les avoir vraisemblablement dépouillés d'une partie de leurs objets de valeur, ont procédé à une réduction des corps sous leurs pas ou peu s'en faut (fig. 26) ${ }^{8}$. Continuant leur avancée, ils ont d'abord rencontré puis suivi le tas de chaux avant de buter contre le mur occidental qu'ils ont perforé en rejetant les moellons derrière eux. La suite de leur trajet ne peut pas être déterminée avec certitude : il est toutefois vraisemblable, en l'absence de toute mise en évidence d'une perforation dans le mur occidental de la pièce N20/3, qu'ils soient sortis des pièces du rez-de-chaussée en suivant le tunnel déjà creusé pour suivre le tuyau de plomb. 
Fig. 26 - Boutique N20. Victimes de l'éruption dont le corps a subi une réduction lors du passage de pillards après l'éruption, en cours de fouille.

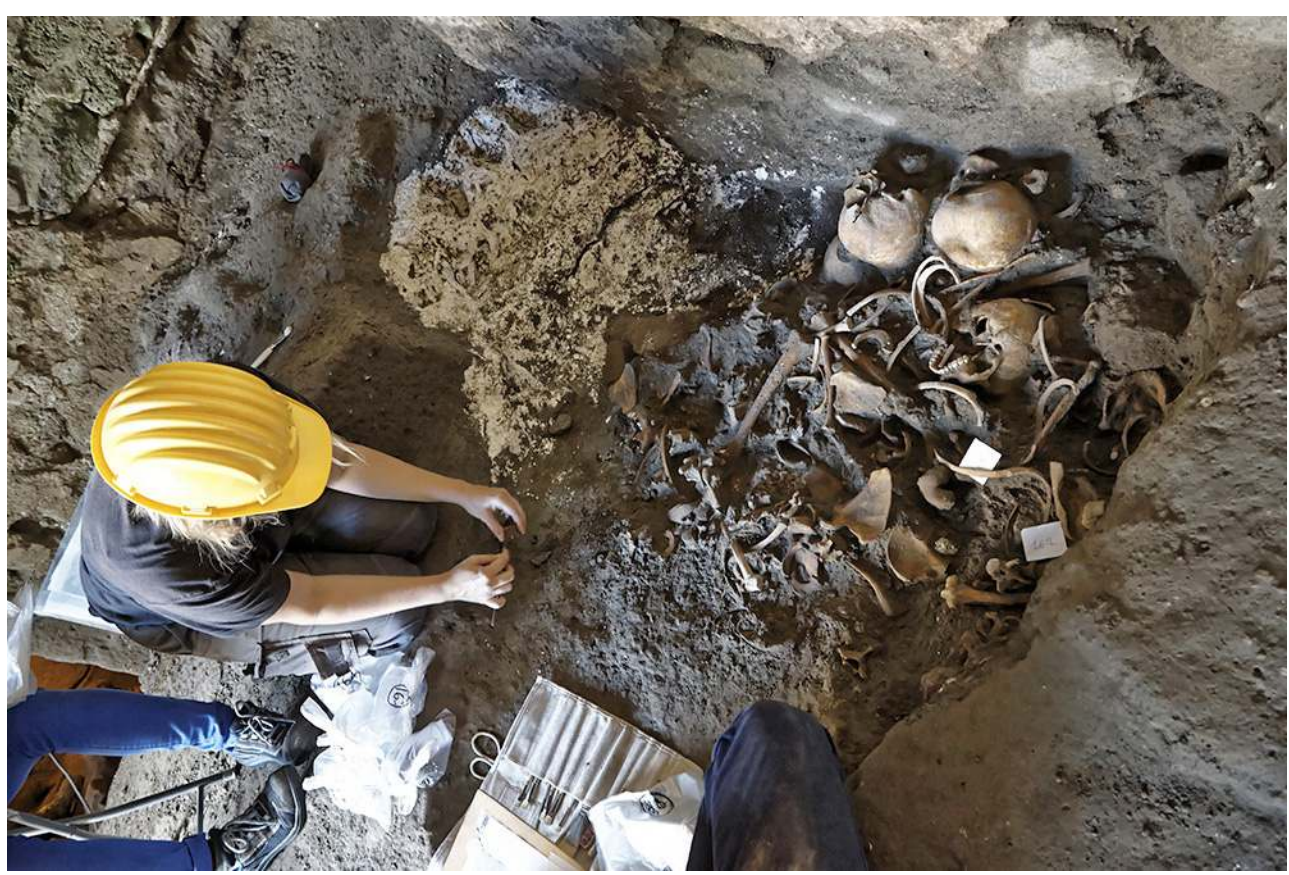

Vue de l'ouest.

Cliché : Fr. Pauvarel.

\section{La pièce 2 de l'espace 30 : l'atelier de potier}

Suite à la fouille de l'atelier de potier entre 2012 et 2015, quelques vérifications s'imposaient dans la pièce $30 / 2$. Un sondage a été rouvert afin d'atteindre les couches laissées en place lors de la dernière campagne ${ }^{9}$. L'objectif était d'atteindre les niveaux de fondation du four situé dans cette pièce et ainsi de chercher à apporter des informations quant à la datation de son installation. Des niveaux antérieurs à son installation et une importante quantité de matériel céramique ont pu être mis au jour : l'étude céramique permettra d'une part d'apporter un terminus quant à la datation de l'installation du four et/ou d'apporter des éléments précieux concernant la production de l'atelier.

\section{La tombe N32a}

Lors de la campagne 2015, deux sépultures avaient été mises au jour ${ }^{10}$. Une troisième avait été aperçue à l'extrémité occidentale du secteur et n'a été fouillée qu'en 2016.

Comme les deux précédentes, il s'agit d'une sépulture constituée de dalles monolithes en calcaire du Sarno posées sur champ, délimitant un espace dans lequel est installé le défunt (fig. 27). Les niveaux en place qui nous sont parvenus permettent d'avancer l'hypothèse que la sépulture a été installée dans le substrat, très probablement avec le creusement d'une fosse dont les limites sont représentées par les blocs formant la sépulture. 
Fig. 27 - Sépulture N32A.

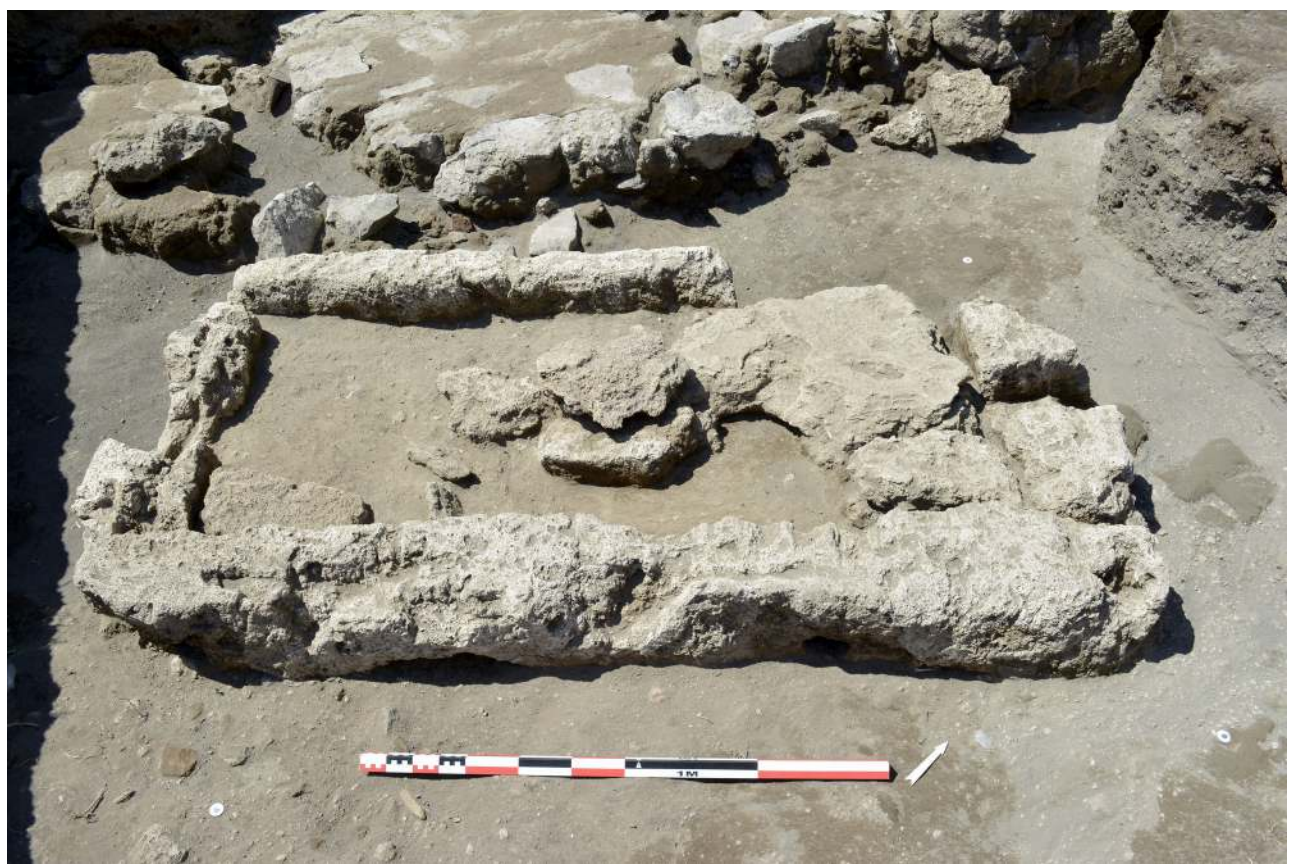

Vue du sud.

Cliché : B. Lemaire-EFR.

En dehors des quelques éléments ayant pu appartenir à une couverture de la tombe, mais remaniés à l'époque moderne/contemporaine, aucune trace du système de fermeture n'a pu être mise en évidence. Cependant, sur les petits côtés de la sépulture, deux encoches traversantes ont pu être observées et pourraient témoigner d'un système de couverture (fig. 28). 
Fig. 28 - Paroi nord de la sépulture avec détail d'une encoche.

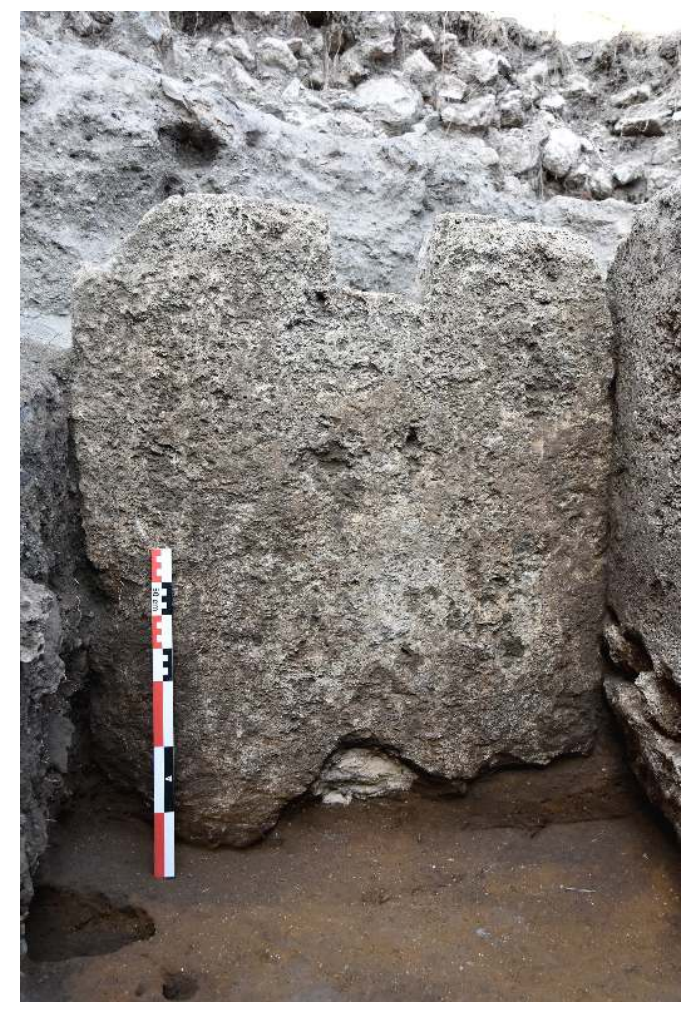

Vue du sud.

Cliché : B. Lemaire-EFR.

Le fond de la sépulture est constitué du substrat creusé par l'installation de la tombe, recouvert d'une fine pellicule blanche. C'est sur ce sol préparé que le défunt a été déposé accompagné de six vases (fig. 29). 
Fig. 29 - Vue zénithale de la sépulture, du défunt et des offrandes.

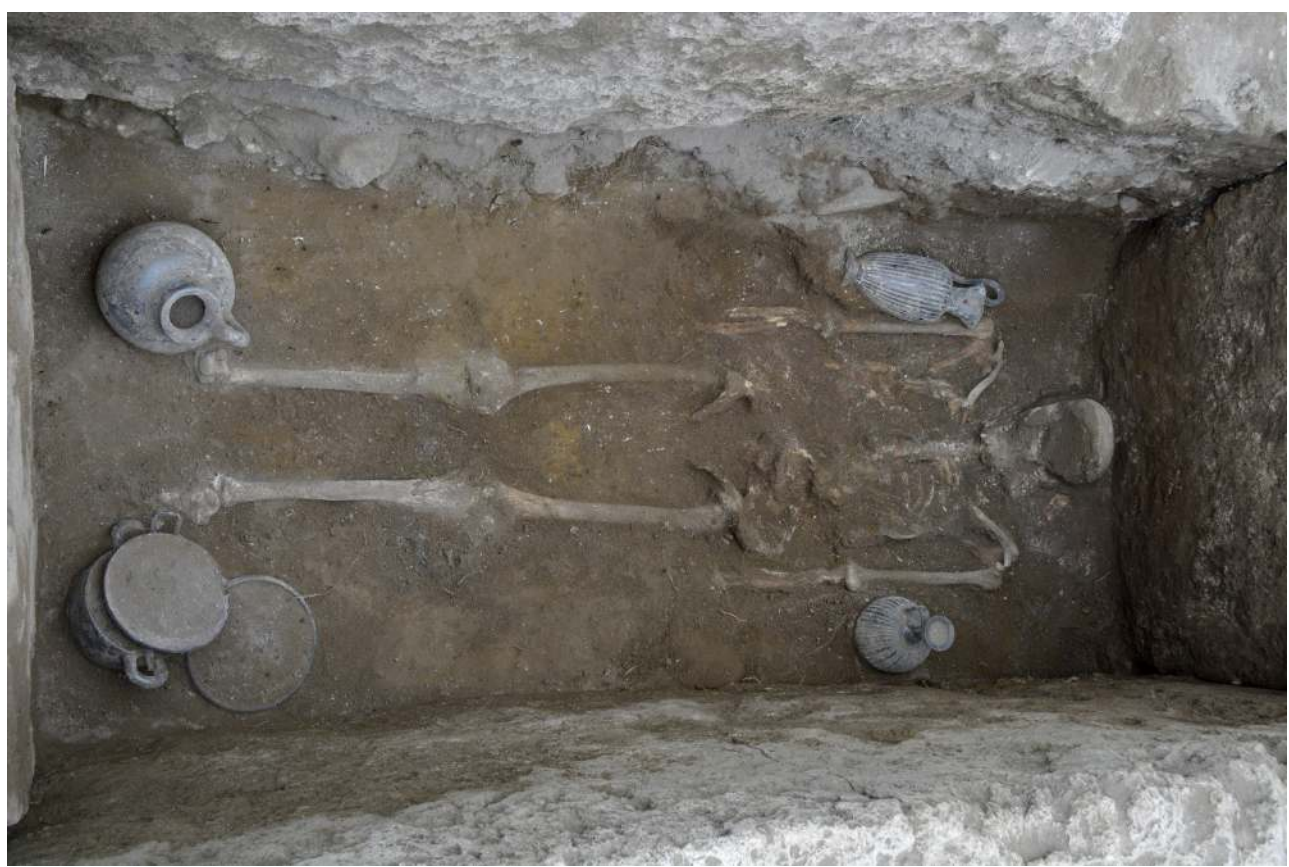

Cliché : B. Lemaire.

La répartition est la suivante: on retrouve une oenochoe en position allongée contre le bras droit, un lécythe en position verticale à proximité du bras gauche, puis trois vases à côté de la jambe gauche: une coupe sur haut pied, une kylix, et un skyphos, tandis qu'à proximité de la jambe droite, se trouvait une cruche. Les datations apportées par un premier examen du mobilier céramique permettent d'avancer l'hypothèse que la sépulture daterait du milieu du IV ${ }^{\mathrm{e}}$ siècle av. n. è. (fig. 30). 
Fig. 30 - Mobilier funéraire de la sépulture.

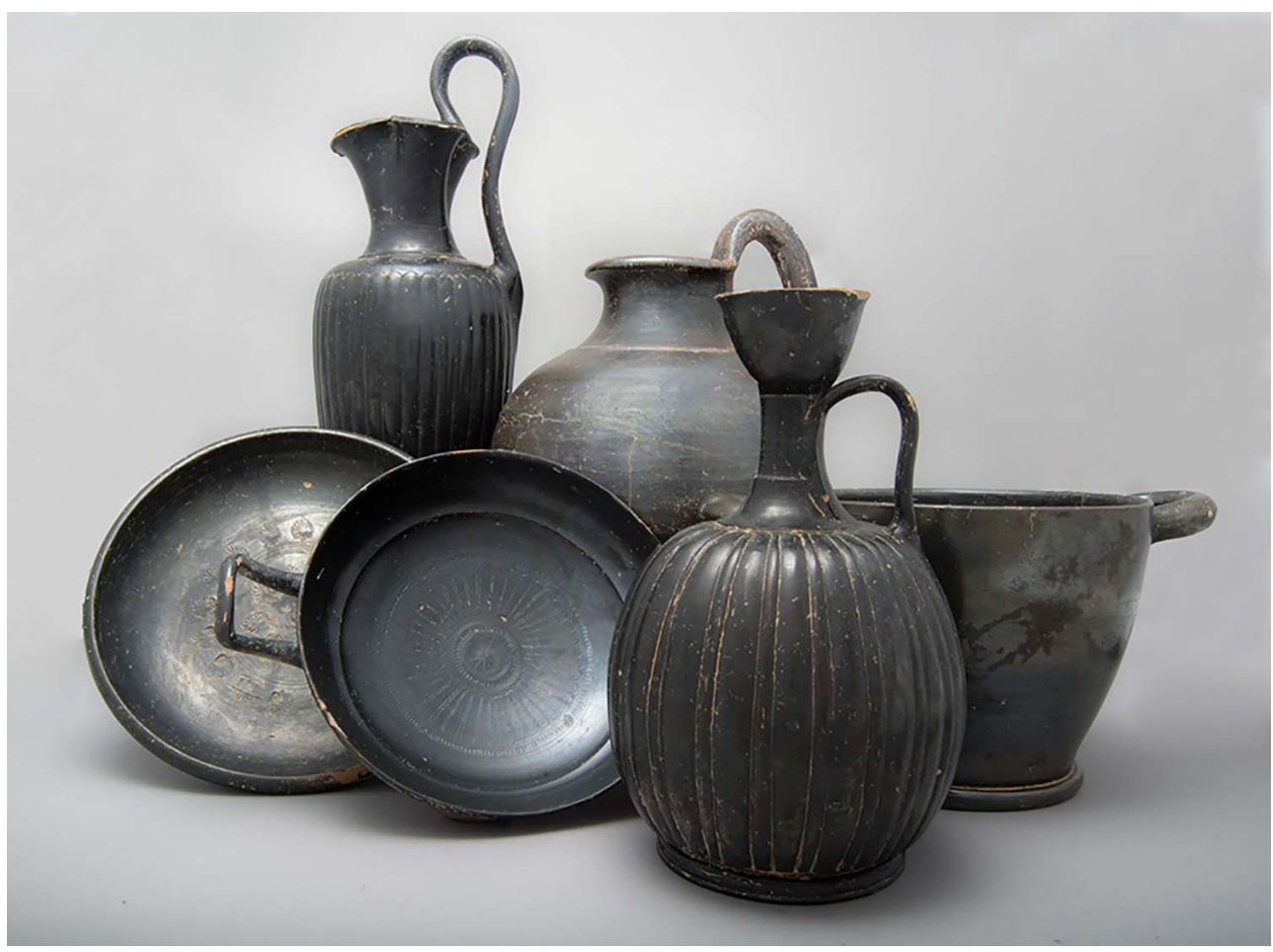

CLICHÉ : C. DURAND (AMU-CNRS-CCJ).

Il s'agit d'une sépulture primaire et individuelle. L'individu est un sujet adulte, probablement de sexe masculin, âgé de plus de 20 ans. Il est déposé sur le dos, la tête au nord-ouest et les pieds au sud-est. Au vu des différents mouvements observés tels que la mise à plat des éléments de côtes conservés, la mise à plat des coxaux et l'effondrement des pieds, la décomposition s'est opérée en espace vide.

L'inhumation, son niveau de dépose, ainsi que les offrandes associées sont recouverts d'un niveau d'infiltrations. Il s'agit d'un niveau de limon argileux compact et hétérogène, de couleur brun clair à jaunâtre (proche du substrat dans lequel s'installe la sépulture). La couche est présente sur l'ensemble de la sépulture. Des inclusions de petits charbons, de traces blanchâtres et de rares fragments de céramiques y ont été observées. Sur ce niveau repose la couverture recouverte à son tour par un remblai moderne voire contemporain.

Comme lors de la précédente campagne, les éléments stratigraphiques attestent que les tombes semblent avoir été dégagées à l'époque moderne ou contemporaine. Un nouvel élément vient renforcer ce fait. Les restes de fixations en ciment moderne d'une plaque, comme on en trouve à l'entrée des boutiques du portique, ont pu être mis en évidence. Il semble donc que ces tombes aient été visibles à un moment donné et portaient un numéro comme tous les autres monuments de Pompéi ${ }^{11}$.

Enfin, à l'extrémité occidentale du secteur, un pan de mur moderne a pu être observé et correspond très probablement à l'effondrement de l'ancien mur d'enceinte du site (fig. 31). 
Fig. 31 - Détail de l'effondrement du mur moderne au premier plan.

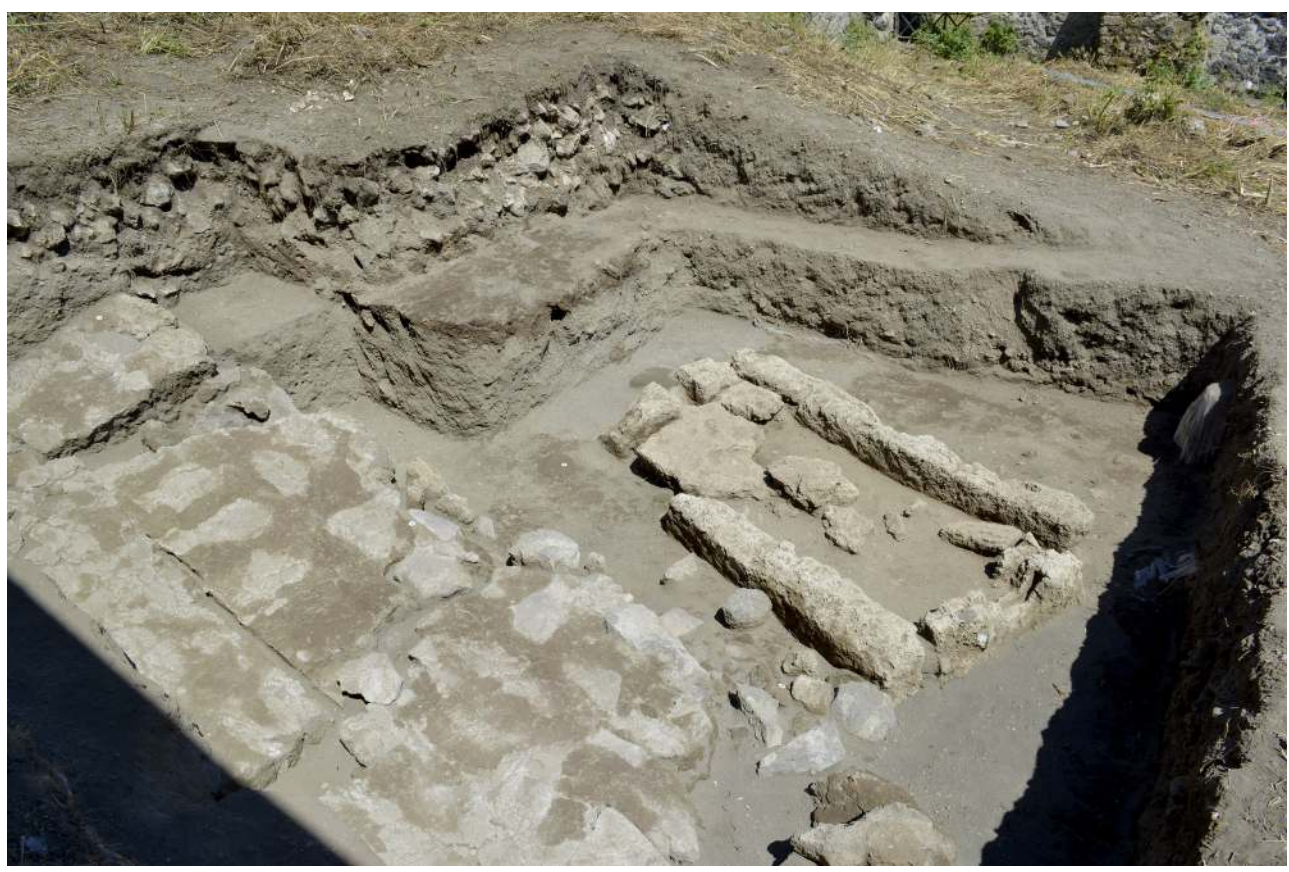

Vu de l'est.

Cliché : B. Lemaire-EFR.

Celui-ci a pu s'effondrer lors des bombardements de 1943, mais l'étude des archives permettra peut-être d'apporter des réponses plus précises.

\section{BIBLIOGRAPHIE}

Adamski - Pernot - Sireix 2011 = F. Adamski, M. Pernot, C. Sireix, Un atelier de production de grands

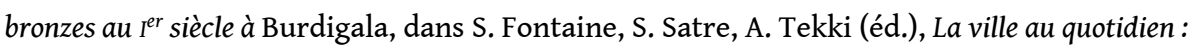
regards croisés sur l'habitat et l'artisanat antiques, Afrique du Nord, Gaule et Italie, Aix-en-Provence, 2011, p. 191-202.

Cavassa et al. 2014 = L. Cavassa, B. Lemaire, G. Chapelin, A. Lacombe, J.-M. Piffeteau, G. Stelo, Pompéi : l'atelier de potier de la via dei Sepolcri, 29, dans Chronique des activités archéologiques de l'École française de Rome [en ligne], 2014, https://cefr.revues.org/1139.

Fiorelli 1875 = G. Fiorelli, Descrizione di Pompei, Naples, 1875 .

Guyard 2003 = L. Guyard (éd.), Le Collège de France (Paris) : du quartier gallo-romain au Quartier latin (I er s. av. J.-C. - XIX ${ }^{e}$ s.), Paris, 2003 (Documents d'archéologie française, 95).

Morel - Chevalley - Castella 2001 = J. Morel, C. Chevalley, D. Castella, La fabrication de grands bronzes à Aventicum : une fosse de coulée dans l'insula 12, dans BPA, 43, 2001, p. 141-162. 
Thomas - Mille - Loiseau $2015=$ N. Thomas, B. Mille, C. Loiseau, Fondre une statue à Vindinum au II siècle, dans S. Raux, V. Brouquier-Reddé, M. Monteil, W. Van Andringa (éd.), Des dieux \& des hommes : cultes et sanctuaires en Sarthe et en Mayenne dans l'antiquité, Le Mans, 2015, p. 182-188.

Varone 1988 = A. Varone, Attività della Soprintendenza, dans RSP, 2, 1988, p. 145-146.

Zanella et al. 2016 = S. Zanella, L. Cavassa, N. Laubry, N. Monteix, B. Lemaire, Pompéi, Porta Ercolano : organisation, gestion et transformations d'une zone suburbaine, dans Chronique des activités archéologiques de l'École française de Rome [en ligne], 2016, https://cefr.revues.org/1581.

Zimmer 1990 = G. Zimmer, Griechische Bronzegusswerkstätten : zur Technologieentwicklung eines antiken Kunsthandwerkes, Mayence, 1990.

\section{NOTES}

1. Par convention, le nord géographique est décalé de $39^{\circ}$ vers l'est. Il s'ensuit que la via dei Sepolcri est ici décrite comme orientée est-ouest, tandis que le les boutiques et ateliers flanquant la villa delle Colonne a mosaico sont considérées comme étant au nord de la voie.

2. Varone 1988, p. 19.

3. Cavassa et al. 2014, § 10-12.

4. Le prélèvement intégral et le tamisage du remplissage du silo n'ont rien donné, en termes de matériel datant ou de contenu abandonné.

5. Il s'agirait alors de la première fosse de coulée pérenne connue dans l'Antiquité. Les autres exemples, qu'ils proviennent du monde grec (Zimmer 1990) ou du monde romain (Morel Chevalley - Castella 2001; Guyard 2003, p. 64-69 ; Adamski - Pernot - Sireix 2011 ; Thomas - Mille - Loiseau 2015) sont des installations temporaires, vouées à des coulées de grands bronzes, généralement associées à des chantiers de construction.

6. L'étude des traces liées au travail du fer a été confiée à Anika Duvauchelle (Musée d'Avenches) et à Gaspard Pagès (CNRS, UMR 5608 Traces).

7. Parmi ces restes, outre de nombreux fragments de bois carbonisé, des os, fragmentaires et parfois travaillés ont été retrouvés au fond de la fosse. Ils paraissent avoir été utilisés comme combustible. D'autres déchets du travail de l'os - non carbonisés - ont été retrouvés dans le comblement de la fosse située au nord-est de la pièce N20/2. L'étude de ces différents lots d'os a été confiée à Anna Rosa Di Nucci.

8. L'étude des restes anthropiques a été confiée à Henri Duday (CNRS) et à Géraldine SachauCarcel (Labex Archimede - UMR 5199 PACEA).

9. Zanella et al. 2016, § 38 .

10. Zanella et al. 2016, § 39-48.

11. Fiorelli 1875 , p. 416 


\section{INDEX}

\section{Index géographique : Pompéi}

institutions École française de Rome, Centre Jean Bérard (USR 3133 CNRS/EFR), Soprintendenza speciale per i beni archeologici di Pompei, Ercolano e Stabia, Collège de France, Centre Camille Jullian (Aix Marseille Univ, CNRS, CCJ, Institut universitaire de France, France), Eveha International, Hadès, Université de Rouen (EA 3831 GRIHS)

\section{AUTEURS}

\section{SANDRA ZANELLA}

Labex Archimede - Université Montpellier III, UMR 5140 Archéologie des Sociétés Méditerranéennes / UMR 8546 AOROC - sandra.zanella[at]univ-montp3.fr

\section{LAETITIA CAVASSA}

Aix Marseille Univ, CNRS, MCC, CCJ, F-13000, Aix-en-Provence, France - cavassa[at]mmsh.univaix.fr

\section{NICOLAS LAUBRY}

Université Paris-Est Créteil, CRHEC (EA 4392) - nicolas.laubry[at]u-pec.fr

\section{NICOLAS MONTEIX}

Université de Rouen, GRHis (EA 3831) - nicolas.monteix[at]univ-rouen.fr

\section{GUILHEM CHAPELIN}

Centre Jean Bérard (USR 3133 CNRS/EFR)

\section{ARNAUD COUTELAS}

Arkemine

\section{AUDREY DELVIGNE RYRKO}

Archéologue indépendante

\section{MÉLANIE ERRERA}

Université Paul-Valéry Montpellier III

\section{LÉA GERARDIN}

Hadès - Bureau d'investigations archéologiques

\section{BASTIEN LEMAIRE}

Université Paul-Valéry Montpellier III, UMR 5140 Archéologie des Sociétés Méditerranéennes 


\section{RAPHAEL MACARIO}

Hadès - Bureau d'investigations archéologiques

\section{FLORIEN ORTIS}

Université Paul-Valéry Montpellier III

\section{VINCENZO PELLEGRINO}

Université Paul-Valéry Montpellier III, UMR 5140 Archéologie des Sociétés Méditerranéennes GÉRALDINE SACHAU-CARCEL

Université Paul-Valéry Montpellier III, UMR 5140 Archéologie des Sociétés Méditerranéennes 